\title{
Multilepton signals of heavier electroweakinos at the LHC
}

\author{
Manimala Chakraborti, ${ }^{a}$ Amitava Datta, ${ }^{b}$ Nabanita Ganguly ${ }^{c}$ and Sujoy Poddar $^{d}$ \\ ${ }^{a}$ Bethe Center for Theoretical Physics 8 Physikalisches Institut der Universität Bonn, \\ Nußallee 12, 53115 Bonn, Germany \\ ${ }^{b}$ Fellow of Indian National Science Academy, \\ Bahadur Shah Zafar Marg, New Delhi — 110002, India \\ ${ }^{c}$ Department of Physics, University of Calcutta, \\ 92 Acharya Prafulla Chandra Road, Kolkata — 700009, India \\ ${ }^{d}$ Department of Physics, Diamond Harbour Women's University, \\ Diamond Harbour Road, Sarisha, South 24 Parganas, West Bengal - 743368, India \\ E-mail: mani.chakraborti@gmail.com, adatta_ju@yahoo.co.in, \\ nabanita.rimpi@gmail.com, sujoy.phy@gmail.com
}

ABSTRACT: As a sequel to our recent paper we examine the phenomenology of the full electroweakino sector of the pMSSM without invoking the ad hoc but often employed assumption that the heavier ones are decoupled. We showcase the importance of the heavier electroweakinos using several generic models with different hierarchies among the slepton and electroweakino masses. We obtain constraints from the LHC $3 l+\not_{T}$ data which are stronger than that for decoupled heavier electroweakinos. Using the additional constraints from the observed dark matter relic density of the universe and the precisely measured anomalous magnetic moment of the muon we determine the allowed parameter space. We then show that novel $m l+\mathbb{E}_{T}$ signatures with $m>3$ may be observed before the next long shut down of the LHC.

KEYwORDS: Supersymmetry Phenomenology

ArXiv EPrint: 1707.04410 


\section{Contents}

1 Introduction 1

2 Models of non-decoupled heavier EWeakinos 3

2.1 The compressed Light Higgsino Heavy Slepton (LHHS) model 5

2.2 The Light Higgsino Heavy Slepton (LHHS) model 5

2.3 The Light Higgsino light Left Slepton (LHLS) model 6

2.4 The Light Mixed light Left Slepton (LMLS) model 6

3 Production and decay modes of EWeakinos in different models $\quad 6$

4 The methodology $\quad 9$

4.1 The constraints 9

$\begin{array}{ll}4.2 \text { The simulation } & 12\end{array}$

$\begin{array}{lll}4.3 & \text { Scanning the parameter space } & 12\end{array}$

5 Constraining models with non-decoupled heavier EWeakinos $\quad 13$

$\begin{array}{ll}5.1 \text { Compressed LHHS model } & 13\end{array}$

$\begin{array}{lll}5.2 & \text { LHHS model } & 15\end{array}$

$\begin{array}{lll}5.3 & \text { LHLS model } & 16\end{array}$

$\begin{array}{lll}5.4 & \text { LMLS model } & 17\end{array}$

$\begin{array}{llr}6 & \text { The multilepton signatures } & \mathbf{1 7}\end{array}$

6.1 The prospective $3 l+\mathbb{E}_{T}$ signal before the next long shut down of the LHC 18

6.2 The prospective $4 l+\mathbb{E}_{T}$ signal 20

6.3 Three same sign and one opposite sign leptons (SS3OS1) $+\mathbb{E}_{T}$ signal 22

$6.45 l+\mathbb{E}_{T}$ signal 24

6.5 Multilepton signals in moderately compressed LHHS models 27

6.6 Discriminating different pMSSM models via multilepton signatures 27

$\begin{array}{llr}7 & \text { Conclusion } & 28\end{array}$

\section{Introduction}

The search for supersymmetry (SUSY) (For reviews on supersymmetry, see, e.g., [1-4]$[5,6])$, the most well motivated extension of the Standard Model (SM) of particle physics, is in progress at the Large Hadron Collider (LHC) for the last few years [7, 8]. Unfortunately the experiments have yielded null results so far leading to limits on the masses of some supersymmetric partners - collectively known as the sparticles. 
In this paper we focus our attention on the electroweak (EW) sector of the supersymmetric standard model. In addition to novel LHC signatures this sector has several other important predictions. It is well known that the sparticles in this sector may explain the origin of the observed dark matter (DM) relic density of the universe [9, 10], for review see, e.g., [11-25]. ${ }^{1}$ In addition light sparticles belonging to this sector may also contribute to the anomalous magnetic moment of the muon $\left(a_{\mu}\right)$ so that the alleged disagreement between this precisely measured quantity $[32,33]$ and the SM prediction (for a review see, e.g., [34]) is significantly reduced due to contributions from virtual sparticles [35]. In this context one must not forget the naturalness criterion ([36-38]-[39]) one of the main motivations for invoking SUSY. ${ }^{2}$ It is well known that the naturalness of a SUSY model crucially depends on the magnitude of the higgsino mass parameter $\mu[1-4]$ which also belongs to the EW sector. The constraints on this parameter emerging from the LHC data and other observables can potentially test the naturalness of the models under consideration.

The masses and other parameters belonging to the EW sector have been constrained by the $3 l+\mathbb{E}_{T}$ searches by the LHC collaborations [42, 43]. However, extracting these limits from the data is by no means straightforward. Ambiguities inevitably arise due to the fact that the SUSY breaking mechanism is yet to be discovered. As a result the masses of a plethora of sparticles and many other important parameters are essentially unknown. In order to simplify the analyses the number of unknown parameters contributing to a particular SUSY signal is reduced by imposing additional assumptions which often turn out to be rather ad hoc in nature. Obviously analyses reducing such ad hoc assumptions, as far as practicable, are desirable for drawing the conclusion on the viability of SUSY, a novel symmetry with many elegant features. Moreover such assumption may obfuscate novel signatures of SUSY which can show up at the LHC in near future as we shall show below.

For example, the limits obtained from the searches for the electroweakinos (EWeakinos), the superpartners of the gauge and Higgs bosons, in the $3 l+\mathbb{E}_{T}$ channel during LHC Run I [42, 43] involved several restrictive assumptions regarding these sparticles. Our phenomenological analyses [44,45] using ATLAS Run I data relaxed some of the above assumptions and showed that the constraints could be significantly weaker. However, all the above and several other recent phenomenological studies [46-50] invoked an ad hoc assumption that only a limited number of relatively light EWeakinos contribute to the $3 l$ signal while the heavier ones are decoupled. In the post LHC era the phenomenology of the heavier Eweakinos was considered in [51, 52] and [53]. However, in [51, 53] all scalar superpartners including the sleptons were assumed to be decoupled. In contrast [52] and this paper consider models in which either the heavier EWeakinos or both lighter and heavier EWeakinos directly decay into on-shell sleptons. These decays are instrumental in enhancing the multilepton signals discussed in the next paragraph.

In [52] it was illustrated that the ATLAS $3 l$ data from Run I was already quite sensitive to the masses of the heavier EWeakinos. Should this signal show up during LHC Run II models with both decoupled and non-decoupled EWeakinos must be included in attempts

\footnotetext{
${ }^{1}$ However co-annihilation of strongly interacting sparticles with the LSP may produce the observed DM in the universe [26, 27]. More recent works can be found in [28-31].

${ }^{2}$ For more recent ones see, e.g., [40, 41].
} 
to decipher the underlying physics. More important, novel $m l+\mathbb{E}_{T}$ with $m>3$, some of which are not viable in models with decoupled heavy EWeakinos, may show up before the next long shutdown of the LHC [52]. Two of these signals were first discussed in [52].

In this paper we extend and complement [52] in several ways. First we make a detailed study of LHC phenomenology in several scenarios briefly studied in [52] using a few benchmark points (BPs) only. Moreover, we delineate the allowed parameter space (APS) of several interesting models taking into account additional constraints like the observed dark matter (DM) relic density of the universe [9, 54], and the precisely measured anomalous magnetic moment of the muon $\left(a_{\mu}\right)[32,33]$ not done in [52]. We also briefly comment on the naturalness ([36-38]-[39]) of the models examined in this paper. Finally, we examine the prospect of observing the $m l+\mathbb{E}_{T}$ signature for $m \geq 3$ before the next long shutdown of the LHC.

In section 2, we briefly describe the models of EWeakinos studied in this paper and earlier works. In section 3, we illustrate the production and decay modes of the heavier EWeakinos with the help of benchmark points. In section 4, we present the methodology followed for obtaining the main results of this paper. In section 5 , we analyse some of the models in section 2 using the constraints discussed in section 4.1 and identify the allowed parameter space in each case. In section 6 , we illustrate the potentially observable $m l+\mathbb{E}_{T}$ signatures for $m \geq 3$ before the next long shutdown of the LHC. Finally, we conclude in section 7 .

\section{Models of non-decoupled heavier EWeakinos}

In the R-parity conserving minimal supersymmetric standard model (MSSM), the EW sector comprises of the following sparticles. The fermionic sparticles are the charginos $\left(\widetilde{\chi}_{j}^{ \pm}, j=1,2\right)$ and the neutralinos $\left(\widetilde{\chi}_{i}^{0}, i=1-4\right)$ - collectively called the EWeakinos. The masses and the compositions of these sparticles are determined by four parameters: the $\mathrm{U}(1)$ gaugino mass parameter $M_{1}$, the $\mathrm{SU}(2)$ gaugino mass parameter $M_{2}$, the higgsino mass parameter $\mu$ and $\tan \beta$ - the ratio of the vacuum expectation values of the two neutral Higgs bosons. If no assumption regarding the SUSY breaking mechanism is invoked the soft breaking masses $M_{1}, M_{2}$ and the superpotential parameter $\mu$ are all independent. Throughout this paper we take $\tan \beta=30$ since relatively large values of this parameter give a better agreement with the $a_{\mu}$ data and ensure that the SM like Higgs boson has the maximum possible mass at the tree level. The indices $i$ and $j$ are arranged in ascending order of the masses. The stable, neutral lightest neutralino $\left(\widetilde{\chi}_{1}^{0}\right)$, which is assumed to be the lightest supersymmetric particle (LSP), is a popular DM candidate.

In the phenomenological MSSM (pMSSM) [55], a simplified version of the MSSM, reasonable assumptions like negligible flavour changing neutral currents and CP violation are invoked to reduce the number of free parameters to 19. In this case the parameters belonging to the EWeakino sector introduced in the last paragraph are assumed to be real and the slepton mass matrices are assumed to be diagonal in the flavour basis. All the observables considered in this paper can be computed in this framework in a straightforward way.

The scalar sparticles are the $L$ and $R$ type sleptons which are superpartners of leptons with left and right chirality. The sneutrinos are the superpartners of the neutrinos. We 
assume L (R)-type sleptons of all flavours to be mass degenerate with a common mass $m_{\widetilde{l}_{L}}\left(m_{\widetilde{l}_{R}}\right)$. Because of the $\mathrm{SU}(2)$ symmetry the sneutrinos are mass degenerate with Lsleptons modulo the D-term contribution. We neglect L-R mixing in the slepton sector. For simplicity we work in the decoupling regime (See e.g., [56]) of the Higgs sector of the MSSM with only one light, SM like Higgs boson, a scenario consistent with all Higgs data collected so far (See, e.g., [57]).

The constraints on the EWeakino masses from the LHC searches are also sensitive to their compositions which are governed by the hierarchy among the parameters $M_{1}, M_{2}$ and $\mu$. Most of the existing analyses revolve around two broad scenarios listed below. ${ }^{3}$

a) The Light Wino (LW) models: they are inspired by the simplified models employed by the LHC collaborations to interpret the $3 l$ data. It is assumed that the lighter EWeakinos $\widetilde{\chi}_{1}^{ \pm}$and $\widetilde{\chi}_{2}^{0}$ are purely wino and nearly mass degenerate while the $\widetilde{\chi}_{1}^{0}$ is bino dominated $([42-44])$.

This scenario can be easily realized in the pMSSM [44]. Here the wino dominated lighter EWeakinos, $\widetilde{\chi}_{1}^{ \pm}$and $\widetilde{\chi}_{2}^{0}$, have nearly degenerate masses governed by the parameter $M_{2}$ while the LSP $\left(\widetilde{\chi}_{1}^{0}\right)$ could be bino dominated with mass controlled by the U(1) gaugino mass parameter $M_{1}\left(M_{1} \ll M_{2}\right)$. The heavier EWeakinos $\left(\widetilde{\chi}_{2}^{ \pm}, \widetilde{\chi}_{3}^{0}\right.$ and $\left.\widetilde{\chi}_{4}^{0}\right)$ are higgsino like with masses approximately equal to $\mu$, where $M_{2}<\mu$. The somewhat ad hoc assumption that these higgsino like sparticles are decoupled requires $M_{2} \ll \mu$. This decoupling can be introduced in numerical computations by setting, e.g., $\mu=2 M_{2}$ [44].

However, computation of the DM relic density immediately reveals that the results are not always consistent with the measured value if the compositions of the EWeakinos are exactly as stated above. For example, a glance at Fig 1b of [44] indicates that these compositions are strictly realized in the parameter space with $m_{\widetilde{\chi}_{1}^{0}} \ll m_{\tilde{\chi}_{1}^{ \pm}}$. On the other hand this parameter space is not consistent with the observed DM relic density of the universe. In fact the only parameter space allowed by the DM relic density constraint (the upper red dotted line in this figure) corresponds to $m_{\widetilde{\chi}_{1}^{0}} \approx m_{\widetilde{\chi}_{1}^{ \pm}}$. In other words the DM constraint is satisfied if the $\widetilde{\chi}_{1}^{0}\left(\widetilde{\chi}_{1}^{ \pm}\right)$though dominantly a bino (wino) has a sizable wino (bino) component in it. Thus consistency with both LHC and DM constraints requires some admixture in the compositions of the EWeakinos. Similar conclusions follow for most of the LW models considered in [44]. Moreover, the light wino models with typically large $\mu$ are also disfavoured by naturalness arguments.

b) The Light Higgsino (LH) models: in this paper, following [45, 52], we mainly consider scenarios with higgsino dominated $\widetilde{\chi}_{1}^{ \pm}, \widetilde{\chi}_{2}^{0}$ and $\widetilde{\chi}_{3}^{0}$ while the LSP is either bino dominated or a bino-higgsino admixture. The three lighter EWeakinos have closely spaced masses governed by $\mu$ while the $\widetilde{\chi}_{1}^{0}$ is either bino dominated with mass controlled by $M_{1}$ or a binohiggsino admixture $\left(M_{1} \lesssim \mu\right)$. The two heavier EWeakinos $\left(\widetilde{\chi}_{2}^{ \pm}\right.$and $\left.\widetilde{\chi}_{4}^{0}\right)$ are wino like with masses approximately equal to $M_{2}$, where $M_{2}>\mu$. However, the choice $M_{2}=2 \mu$ in [45] effectively decouples these sparticles. In summary we do not invoke the ad hoc assumption that the heavier EWeakinos are decoupled (i.e., $\mu \gg M_{2}$ in case a) and $M_{2} \gg \mu$ in case b)).

In all models the trilepton rates also depend sensitively on the hierarchy among the slepton and EWeakino masses. If the sleptons are lighter (heavier) than $\widetilde{\chi}_{1}^{ \pm}$and $\widetilde{\chi}_{2}^{0}$, the

\footnotetext{
${ }^{3}$ We shall, however, briefly comment on other models as well.
} 
leptonic Branching Ratios (BRs) of these EWeakinos are typically large (small) yielding stronger (weaker) limits.

It may be recalled that the strongest lower limit $m_{\widetilde{\chi}_{1}^{ \pm}}>800 \mathrm{GeV}$ for negligible LSP mass [42] is obtained in the Light Wino with Light Left Slepton (LWLS) model. Here all L-sleptons masses are fixed at the arithmetic mean of $m_{\widetilde{\chi}_{1}^{0}}$ and $m_{\widetilde{\chi}_{1}^{ \pm}}$which enhances the leptonic BRs of the decaying EWeakinos. All R-sleptons are assumed to be decoupled. In view of the above lower limit the heavier EWeakino masses are automatically restricted to be rather high so that they cannot significantly enhance the trilepton and other signals. If the L-sleptons are heavier than $\widetilde{\chi}_{1}^{ \pm}$, the bounds on $m_{\widetilde{\chi}_{1}^{ \pm}}$are much weaker $(\approx 350 \mathrm{GeV}$ for negligible LSP masses). However, the production cross sections of the higgsino dominated heavier EWeakino pairs are in general suppressed as discussed in [45]. Thus the cross sections of all multilepton signals from their cascade decays tend to be small. It is worth noting that the wino like heavier EWeakinos in the LH model have relatively large crosssections which to some extent compensate the suppression due to their large masses.

The above discussions motivate us to primarily focus on the LH type models with occasional comments on the LW models.

\subsection{The compressed Light Higgsino Heavy Slepton (LHHS) model}

In the compressed LHHS model first considered in [52], $M_{1} \simeq \mu$ with $M_{2}>\mu$. This choice of parameters leads to a compressed lighter EWeakino spectrum where $\widetilde{\chi}_{1}^{0}, \widetilde{\chi}_{2}^{0}, \widetilde{\chi}_{3}^{0}$ and $\widetilde{\chi}_{1}^{ \pm}$ are approximately mass degenerate and each has significant bino and higgsino components. The masses of the wino dominated heavier EWeakinos are determined by the free parameter $M_{2}$. Here the common slepton mass is set to be $m_{\tilde{l}_{L}}=m_{\tilde{l}_{R}}=\left(m_{\tilde{\chi}_{1}^{ \pm}}+m_{\tilde{\chi}_{2}^{ \pm}}\right) / 2$ so that sleptons are always heavier than lighter EWeakinos.

It is well known that if the LSP is a bino-higgsino admixture the DM relic density constraint can be satisfied [58]-[40]. In addition this model is worth studying from the point of view of naturalness since $\mu$ is necessarily small. On the other hand since $\widetilde{\chi}_{1}^{ \pm}, \widetilde{\chi}_{2}^{0}$ and $\widetilde{\chi}_{3}^{0}$ are nearly mass degenerate with $\tilde{\chi}_{1}^{0}$, any signal stemming from the lighter EWeakino sector essentially consists of soft particles in the final state and are hard to detect. This tension is relaxed if the heavier EWeakinos $\left(\widetilde{\chi}_{2}^{ \pm}\right.$and $\left.\widetilde{\chi}_{4}^{0}\right)$ are relatively light. Observable multilepton signals from their cascade decays indeed look promising [52]. This issue will be taken up in further details in the next section and section 5. In the rest of this paper this model will be simply referred to as the compressed model.

\subsection{The Light Higgsino Heavy Slepton (LHHS) model}

In this class of models [45] the mass parameters $\mu<M_{2}$ whereas $M_{1}$ is taken to be the lightest. The mass of LSP is determined by $M_{1}$ and it is bino dominated. $\widetilde{\chi}_{1}^{ \pm}, \widetilde{\chi}_{2}^{0}$ and $\widetilde{\chi}_{3}^{0}$ are higgsino dominated with closely spaced masses controlled by $\mu$. The wino like heavier EWeakinos - $\widetilde{\chi}_{2}^{ \pm}$and $\widetilde{\chi}_{4}^{0}$ - have masses controlled by $M_{2}$. For numerical results we set $M_{2}=1.5 \mu$. Both left and right sleptons masses are taken to be midway between $m_{\tilde{\chi}_{1}^{ \pm}}$ and $m_{\tilde{\chi}_{2}^{ \pm}}$. Consequently, only heavier EWeakinos can decay directly into sleptons with significant BRs. Although $\widetilde{\chi}_{2}^{ \pm}, \widetilde{\chi}_{4}^{0}$ have suppressed production cross-sections compared to 
the lighter EWeakinos, they have moderately large lepton yield thanks to their cascade decays involving sleptons, lighter EWeakinos, $W$ and $Z$ bosons all of which can decay leptonically. One can, therefore, expect to get sizable multilepton ( $4 l$ and $5 l$ ) signals from their cascade decays in additional to the conventional $3 l$ final states.

\subsection{The Light Higgsino light Left Slepton (LHLS) model}

This model is the same as the previous one except that the masses of the L-sleptons are chosen to lie midway between $m_{\widetilde{\chi}_{1}^{0}}$ and $m_{\widetilde{\chi}_{2}^{0}} / m_{\widetilde{\chi}_{1}^{ \pm}}$whereas R-slepton masses are set at $2 \mathrm{TeV}$. Due to leptons coming from both lighter and heavier EWeakinos, the bounds on $m_{\widetilde{\chi}_{1}^{ \pm}}$or $m_{\widetilde{\chi}_{2}^{0}}$ get stronger. Moreover, multilepton signals are also copiously produced.

\subsection{The Light Mixed light Left Slepton (LMLS) model}

In all mixed models EWeakinos except the LSP are admixtures of higgsino and wino components and have closely spaced masses (i.e., $\mu \simeq M_{2}$ ). The LSP is bino dominated with mass controlled by $M_{1}$. In the LMLS model the left sleptons masses are kept midway between $\widetilde{\chi}_{1}^{0}$ and $\widetilde{\chi}_{1}^{ \pm}$whereas right sleptons are decoupled with masses $\simeq 2 \mathrm{TeV}$.

\section{Production and decay modes of EWeakinos in different models}

Since the production and decay modes of the heavier EWeakinos in models with light sleptons have not been discussed in recent literature we discuss their main features in this section. ${ }^{4}$ It has already been pointed out that the production cross-section of lighter EWeakino pairs are quite sensitive to their compositions (see table 2 of [45]). For fixed $m_{\widetilde{\chi}_{1}^{0}}$ and $m_{\tilde{\chi}_{1}^{ \pm}}$, it is largest in the wino model, smallest in the higgsino model and intermediate in case of the mixed scenario. This result can be readily generalised to the heavy EWeakinos. Naturally the production cross section of $\widetilde{\chi}_{2}^{ \pm}-\widetilde{\chi}_{4}^{0}$ pair in the Higgsino model is suppressed by their large masses. However, this suppression is to some extent compensated since they are wino like. In contrast the production cross sections of the lighter EWeakinos suffer suppression due to their higgsino like composition. At the production level the lighter and heavier EWeakino yields could, therefore, be quite competitive.

In table 1 we present the cross-sections of different EWeakino pairs for representative points in models described in the previous section. The benchmark points $\mathrm{P} 1-\mathrm{P} 4$ correspond to the compressed, LHHS, LHLS and LMLS models respectively. The total production cross section of only lighter EWeakino pairs (all EWeakino pairs involving at least one heavy EWeakino) is denoted by $\sigma(p p \rightarrow$ LEWs $)(\sigma(p p \rightarrow$ HEWs $))$. Comparing the two cross-sections it follows that in all cases the heavier EWeakinos in non-decoupled scenarios significantly contribute to the total EWeakino production in spite of their large masses.

In table 2 we present the BRs of all EWeakinos in scenarios $\mathrm{P} 1-\mathrm{P} 4$. In table 3 we have shown the effective cross-section defined as $(\sigma \times \mathrm{BR})_{m l}$ of multilepton channels with $\mathrm{m}=$ $3,4,5$. Here LEW (HEW) refers to the contribution of lighter EWeakino pairs only (pairs with at least one heavier EWeakino) to a particular signal.

\footnotetext{
${ }^{4}$ In ref. [51] they have been discussed in models with decoupled sleptons.
} 


\begin{tabular}{|c|c|c|c|c|}
\hline \multirow{2}{*}{$\begin{array}{c}\text { Masses } \\
\text { and }\end{array}$} & \multicolumn{4}{|c|}{ Models } \\
\cline { 2 - 5 } Cross-sections & P1 & P2 & P3 & P4 \\
(Compressed) & (LHHS) & (LHLS) & (LMLS) \\
\hline$M_{1}$ & 186 & 105 & 249 & 321 \\
$\mu$ & 191 & 270 & 300 & 401 \\
$M_{2}$ & 350 & 405 & 450 & 382 \\
$m_{\widetilde{\chi}_{1}^{0}}$ & 151 & 100 & 231 & 304 \\
$m_{\widetilde{\chi}_{2}^{0}}$ & 198 & 262 & 304 & 359 \\
$m_{\widetilde{\chi}_{3}^{0}}$ & 213 & 281 & 311 & 412 \\
$m_{\widetilde{\chi}_{4}^{0}}$ & 389 & 447 & 491 & 467 \\
$m_{\widetilde{\chi}_{1}^{ \pm}}$ & 178 & 260 & 291 & 350 \\
$m_{\widetilde{\chi}_{2}^{ \pm}}$ & 389 & 447 & 491 & 465 \\
\hline$\sigma(p p \rightarrow$ LEWs $)$ & 621.9 & 299.5 & 165.8 & 72.94 \\
$\sigma(p p \rightarrow$ HEWs $)$ & 147.1 & 81.4 & 52.0 & 83.95 \\
$\sigma_{\text {tot }}$ & 768.9 & 380.9 & 217.8 & 156.9 \\
\hline
\end{tabular}

Table 1. Mass parameters, physical masses and production cross-sections of EWeakinos for four representative points in different models introduced in section 2. See text for the details. All masses and mass parameters are in GeV. Cross-sections are in $f b$.

In the compressed model (P1), $\sigma$ is much larger for lighter EWeakinos than that for the heavier EWeakinos as expected. But since the sleptons are heavier than the lighter EWeakinos the latter cannot decay directly into sleptons which eventually yields leptonic states with large BRs. However, leptonic decays mediated by virtual $W$ or $Z$ bosons are allowed. Small leptonic BR of the gauge bosons suppresses the leptonic signals if only the lighter EWeakinos are considered. The situation, however, completely changes if one takes into consideration the contributions of heavier EWeakino decays. They can decay either directly into sleptons or leptons can come from lighter EWeakino, $W$ or $Z$ mediated processes. This enhances the multilepton signals quite a bit. This is illustrated in table 3 where we have shown the effective cross-section for different multilepton channels. In the compressed model the leptonic decays of the lighter EWeakinos inevitably lead to soft leptons because of the small energy release. The apparently non-vanishing cross-sections in table 3 are drastically suppressed when appropriate cuts requiring hard leptons in the signal are imposed. This will be discussed in details in a later section.

Next we come to the LHHS model (P2). Again the production cross-section of the lighter EWeakinos dominate over that of the heavier ones. However, heavier EWeakinos have larger leptonic BRs for reasons discussed in the last paragraph. From table 3, one can see the relative contributions of lighter and heavier EWeakinos to multilepton signals. For the $3 l+\mathbb{E}_{T}$ channel both of them have non-negligible contributions although bulk of the events come from the heavier ones. However, the heavier EWeakino contributions to $4 l$ and especially $5 l$ signals are much larger. 


\begin{tabular}{|c|c|c|c|c|c|c|c|c|c|}
\hline \multirow{2}{*}{$\begin{array}{l}\text { Decay } \\
\text { Modes }\end{array}$} & \multicolumn{4}{|c|}{ Branching Ratio } & \multirow{2}{*}{$\begin{array}{l}\text { Decay } \\
\text { Modes }\end{array}$} & \multicolumn{4}{|c|}{ Branching Ratio } \\
\hline & $\mathrm{P} 1$ & $\mathrm{P} 2$ & P3 & $\mathrm{P} 4$ & & $\mathrm{P} 1$ & $\mathrm{P} 2$ & P3 & $\mathrm{P} 4$ \\
\hline$\tilde{\chi}_{2}^{ \pm} \rightarrow \widetilde{\nu} l^{ \pm}$ & 0.17 & 0.14 & 0.24 & 0.24 & $\widetilde{\chi}_{4}^{0} \rightarrow \widetilde{\chi}_{i}^{0} Z$ & 0.12 & 0.11 & 0.06 & - \\
\hline$\rightarrow \widetilde{\nu}_{\tau_{1}} \tau^{ \pm}$ & 0.08 & 0.07 & 0.12 & 0.15 & $\rightarrow \widetilde{\chi}_{1}^{ \pm} W^{\mp}$ & 0.30 & 0.34 & 0.17 & 0.24 \\
\hline$\rightarrow \tilde{l}_{L}^{ \pm} \nu$ & 0.16 & 0.14 & 0.24 & 0.28 & $\rightarrow \widetilde{\chi}_{i}^{0} h$ & 0.09 & 0.10 & 0.05 & 0.01 \\
\hline$\rightarrow \tilde{\tau}_{1,2}^{ \pm} \nu_{\tau}$ & 0.08 & 0.07 & 0.12 & 0.14 & $\rightarrow \tilde{l}_{L}^{ \pm} l^{\mp}$ & 0.14 & 0.11 & 0.22 & 0.16 \\
\hline$\rightarrow \widetilde{\chi}_{1}^{ \pm} Z$ & 0.14 & 0.16 & 0.08 & 0.08 & $\rightarrow \tilde{\tau}_{1,2}^{ \pm} \tau^{\mp}$ & 0.07 & 0.06 & 0.11 & 0.12 \\
\hline$\rightarrow \widetilde{\chi}_{i}^{0} W^{ \pm}$ & 0.27 & 0.31 & 0.15 & 0.11 & $\rightarrow \widetilde{\nu} \nu$ & 0.28 & 0.24 & 0.39 & 0.47 \\
\hline$\rightarrow \tilde{\chi}_{1}^{ \pm} h$ & 0.09 & 0.10 & 0.17 & - & & & & & \\
\hline$\tilde{\chi}_{1}^{ \pm} \rightarrow \tilde{\chi}_{1}^{0} q \overline{q^{\prime}}$ & 0.66 & - & - & - & $\tilde{\chi}_{3}^{0} \rightarrow \widetilde{\chi}_{1}^{0} q \bar{q}$ & 0.02 & - & - & - \\
\hline$\rightarrow \widetilde{\chi}_{1}^{0} l^{ \pm} \nu$ & 0.22 & - & - & & $\rightarrow \widetilde{\chi}_{1}^{0} l^{+} l^{-}$ & 0.02 & - & - & \\
\hline$\rightarrow \widetilde{\chi}_{1}^{0} \tau^{ \pm} \nu_{\tau}$ & 0.11 & - & - & & $\rightarrow \widetilde{\chi}_{1}^{0} \tau^{+} \tau^{-}$ & 0.03 & - & - & - \\
\hline$\rightarrow \widetilde{\chi}_{1}^{0} W^{ \pm}$ & - & 1.0 & - & & $\rightarrow \widetilde{\chi}_{1}^{0} \nu \bar{\nu}$ & 0.02 & - & - & - \\
\hline$\rightarrow \widetilde{\nu} l^{ \pm}$ & - & - & 0.36 & 0.45 & $\rightarrow \widetilde{\chi}_{1}^{ \pm} q \bar{q}$ & 0.60 & - & - & - \\
\hline$\rightarrow \widetilde{\nu}_{\tau_{1}} \tau^{ \pm}$ & - & - & 0.52 & 0.28 & $\rightarrow \tilde{\chi}_{1}^{ \pm} \nu l^{\mp}$ & 0.20 & - & - & - \\
\hline$\rightarrow \tilde{l}_{L}^{ \pm} \nu$ & - & - & 0.08 & 0.18 & $\rightarrow \widetilde{\chi}_{1}^{ \pm} \nu_{\tau} \tau^{\mp}$ & 0.10 & - & - & - \\
\hline$\rightarrow \tilde{\tau}_{1}^{ \pm} \nu_{\tau}$ & - & - & 0.04 & 0.09 & $\rightarrow \widetilde{\chi}_{i}^{0} Z$ & - & 0.91 & - & 0.44 \\
\hline$\widetilde{\chi}_{2}^{0} \rightarrow \widetilde{\chi}_{1}^{0} q \bar{q}$ & 0.63 & - & - & - & $\rightarrow \widetilde{\chi}_{i}^{0} h$ & - & 0.09 & - & - \\
\hline$\rightarrow \widetilde{\chi}_{1}^{0} l^{+} l^{-}$ & 0.06 & - & - & - & $\rightarrow \tilde{l}_{L}^{ \pm} l^{\mp}$ & - & - & 0.01 & - \\
\hline$\rightarrow \widetilde{\chi}_{1}^{0} \tau^{+} \tau^{-}$ & 0.04 & - & - & - & $\rightarrow \tilde{\tau}_{1}^{ \pm} \tau^{\mp}$ & - & - & 0.84 & 0.49 \\
\hline$\rightarrow \widetilde{\chi}_{1}^{0} \nu \bar{\nu}$ & 0.20 & - & - & - & $\rightarrow \widetilde{\nu} \nu$ & - & - & 0.15 & 0.06 \\
\hline$\rightarrow \widetilde{\chi}_{1}^{0} Z$ & - & 0.28 & - & - & $\rightarrow \widetilde{\chi}_{1}^{ \pm} W^{\mp}$ & - & - & - & - \\
\hline$\rightarrow \widetilde{\chi}_{1}^{0} h$ & - & 0.72 & - & - & - & & & & \\
\hline$\rightarrow \tilde{l}_{L}^{ \pm} l^{\mp}$ & - & - & 0.55 & 0.45 & & & & & \\
\hline$\rightarrow \tilde{\tau}_{1}^{ \pm} \tau^{\mp}$ & - & - & 0.45 & 0.26 & & & & & \\
\hline$\rightarrow \widetilde{\nu} \nu$ & - & - & - & 0.29 & & & & & \\
\hline
\end{tabular}

Table 2. Different decay modes of EWeakinos along with their BRs for four representative points as given in table 1.

For the LHLS (P3) and LMLS (P4), the $3 l$ signal is dominated by the lighter EWeakino contributions. However, the $4 l$ and $5 l$ signals are essentially due to the heavier ones.

We conclude this section with the important message that the multilepton signals in the compressed scenario significantly depend on heavier EWeakinos. For other models although both heavy and light EWeakinos can contribute significantly to $3 l+\mathbb{E}_{T}$, final states with more than three leptons essentially come from the heavier ones. 


\begin{tabular}{|c|c|c|c|c|}
\hline$(\sigma \times \mathrm{BR})_{3 l}$ & $\mathrm{P} 1$ & $\mathrm{P} 2$ & $\mathrm{P} 3$ & $\mathrm{P} 4$ \\
\hline LEWs & 9.36 & 2.41 & 18.25 & 20.5 \\
\hline HEWs & 64.2 & 4.85 & 6.23 & 6.46 \\
\hline$(\sigma \times \mathrm{BR})_{4 l}$ & $\mathrm{P} 1$ & $\mathrm{P} 2$ & $\mathrm{P} 3$ & $\mathrm{P} 4$ \\
\hline LEWs & 0.212 & 0.113 & 0.116 & - \\
\hline HEWs & 20.2 & 0.764 & 0.661 & 0.725 \\
\hline$(\sigma \times \mathrm{BR})_{5 l}$ & $\mathrm{P} 1$ & $\mathrm{P} 2$ & $\mathrm{P} 3$ & $\mathrm{P} 4$ \\
\hline LEWs & - & 0.008 & - & - \\
\hline HEWs & 4.81 & 0.134 & 0.137 & 0.118 \\
\hline
\end{tabular}

Table 3. Effective cross-sections, namely $\sigma \times B R$, for three different signals $-3 l, 4 l$ and $5 l$ for four representative points in table 1 . See text for the details. Cross-sections are in $f b$.

\section{The methodology}

In this section we briefly describe the procedure for constraining the models presented in section 2. We also outline the generator level simulation of different LHC signals using PYTHIA (v6.428) [59] and the methods for scanning the parameter space.

\subsection{The constraints}

We have used three major constraints involving relatively small theoretical/experimental uncertainties as listed below.

- We use the ATLAS trilepton data [42] on $\widetilde{\chi}_{1}^{ \pm}-\widetilde{\chi}_{2}^{0}$ searches from LHC Run I. The correlated constraints on LSP and slepton masses as given by the ATLAS slepton search data [60] are also taken into account in models with light sleptons. We also require the lighter Higgs boson mass $m_{h}$ to be in the interval $122<m_{h}<128 \mathrm{GeV}$ around a central value of $125 \mathrm{GeV}[67,68]$. This is achieved by choosing judiciously the third generation trilinear soft breaking term $-A_{t}$, the CP odd Higgs mass $-M_{A}$ and the lighter top squark mass which is chosen to be $1.5 \mathrm{TeV}$. The window of $3 \mathrm{GeV}$ mainly reflects the theoretical uncertainty [69-74] in computing the Higgs mass in a typical SUSY scenario. The heavier Higgs bosons are assumed to be decoupled. It may be recalled that the BRs of the unstable EWeakinos depend on $m_{h}$.

- The precise measurement of the muon anomalous magnetic moment $\left(a_{\mu}=\right.$ $\left.\frac{1}{2}(g-2)_{\mu}\right)[32,33]$ plays an important role in studying new physics. The experimental value of $a_{\mu}$ denoted by $a_{\mu}^{e x p}$ differs significantly from the SM prediction $a_{\mu}^{S M}$ (see, e.g., [34]). This large deviation strongly hints for new physics beyond SM. There are three parts in $a_{\mu}^{S M}$ - a part from pure quantum electrodynamics, a part from EW contributions and the hadronic contributions. The SUSY contribution to $a_{\mu}$, namely $a_{\mu}^{S U S Y}$ becomes large when the charginos, neutralinos and smuons are rela- 
tively light $[35]^{5}$ and it scales with $\tan \beta$. Thus one can constrain the SUSY parameter space with the measured upper and lower bounds on $\Delta a_{\mu}=a_{\mu}^{e x p}-a_{\mu}^{S M}$ given by [35]:

$$
\Delta a_{\mu}=a_{\mu}^{e x p}-a_{\mu}^{S M}=(29.3 \pm 9.0) \times 10^{-10}
$$

The computation of $a_{\mu}^{S U S Y}$ may be found in refs. [75-80]. It should be noted that the Higgs mass at $125 \mathrm{GeV}$ and stringent lower bounds on the masses of the strong sparticles from LHC data strongly disfavour the models like mSUGRA with strong assumptions on soft breaking parameters in the light of $a_{\mu}$ data [81, 82]. However, non-universal gaugino mass models can still resolve the $a_{\mu}$ anomaly within the said range of $\Delta a_{\mu}$ [83-86]. This sensitivity shows that the $a_{\mu}$ data can indeed constrain the slepton and EWeakino masses which are free parameters in the pMSSM.

Since the SM is consistent with the measured $a_{\mu}$ at the $3 \sigma$ level we require that the models under scrutiny yield a better agreement, say, at the level of $\lesssim 2 \sigma$.

- In this analysis we also impose the constraint from the measured DM relic density of the universe $[9,54]$. The $3 \sigma$ limit which we use in this work is:

$$
0.092<\Omega_{\tilde{\chi}} h^{2}<0.138
$$

Apparently this limit is significantly relaxed than the latest experimental limit $0.1199 \pm 0.0022[10]$. This is due to the fact that the above range includes, in addition to the experimental errors, estimated theoretical errors discussed in the literature [87]-[88]. A range similar to the above has been used, e.g., in [89]. Recently it has also been noted that QCD corrections to neutralino annihilation and coannihilation channels involving quarks may introduce sizable uncertainties due to the choice of the QCD scale [90]. In view of the above discussions a more conservative handling of the constraint seems to be justified.

There are other measurements related to DM which are often used to constrain the EWeakino sector of the pMSSM. However, theoretical and experimental uncertainties make them less stringent compared to the one presented above. In the following we briefly summarize them (see the references given below) and indicate how we have treated them in our analysis.

The direct detection experiments measure the DM - nucleon scattering [91] cross sections. Since no scattering has been observed many models of DM have been constrained. It should be borne in mind that the theoretical prediction for the spin independent scattering cross-section $\left(\sigma_{S I}\right)$ crucially depends on the value of the DM relic density $\left(\rho_{E}\right)$ at the detector (i.e., in the neighbourhood of the earth). There are standard astrophysical methods for measuring the local density of DM $\left(\rho_{L}\right)$ which is an average over a volume having a radius of typically a few hundred parsecs (pcs) with the sun at the center [92]. This volume though cosmologically small is huge in the terrestrial length scale. The measured central values of $\rho_{L}$ lies in the range $0.023-0.85 \mathrm{GeV} p c^{-3}$ (see table 4 of [92]). Due

\footnotetext{
${ }^{5}$ For more recent ones see, e.g., [61-66].
} 
to large errors the measured values are comparable with much smaller $\rho_{L}$. However, there is an even bigger source of uncertainty. Getting $\rho_{E}$ from $\rho_{L}$ involves an extrapolation over many orders of magnitudes which is mainly done by simulation. According to [92] the Dark Matter Only (DMO) simulations indicate that $\rho_{E}$ and $\rho_{L}$ may not be very different. The situation, however, is further complicated by the presence of significant amount of ordinary baryonic matter in the solar system and its possible impact on $\rho_{E}$. According to [92] the present techniques cannot predict a reliable $\rho_{E}$. On the other hand the global measure of dark matter density $\rho_{G}$ obtained from the rotation curve of our galaxy typically have smaller errors (see table 4 [92]). But they are based on the strong assumption that the galactic halo is spherically symmetric which may not be realistic [92].

It is well known that there is another way of evading the direct detection limits. For a mixed DM, which is the case for most of the models studied in this paper, it is possible that $\widetilde{\chi}_{1}^{0}-\widetilde{\chi}_{1}^{0}-h$ coupling is significantly suppressed in certain regions of the parameter space (the so called 'blind spots') due to cancellation between different contributions to this coupling [93]. As a result the theoretical prediction for $\sigma^{S I}$ may be further suppressed making it compatible with the direct detection data. In this paper, however, we do not examine the implications of the blind spots numerically.

There are other theoretical/experimental uncertainties in $\sigma^{S I}$ (e.g., the uncertainties in the form factors of LSP - quark scattering) as discussed in section 2.3 of [44] where reference to the original works may be found. Taking all these into account the total uncertainty in the upper limit of the DM - nucleon cross-section could be one order of magnitude or even larger. The spin independent DM - nucleon scattering cross-section $\sigma^{S I}$ has been computed in several LH models [45]. It was argued that the models studied were compatible with the then LUX data [94] on the upper bound on $\sigma^{S I}$ as a function of the DM mass provided allowance was made for the large uncertainties discussed above. However more recent LUX data ([91]) have imposed much stronger constraints on $\sigma^{S I}$. These data taken at its face value impose strong lower limits on the DM mass $\left(m_{\widetilde{\chi}_{1}^{0}}\right)$ which would make some models considered in this paper uninteresting in the context of the ongoing LHC searches. We shall come back to this issue in the next section when we consider different models.

There are interesting attempts to link several anomalies in astrophysical observations with annihilation of DM (see e.g., [95]-[96]). Many of the reported signals are not free from ambiguities because of the uncertainties in the estimation of the astrophysics backgrounds. In addition some of the reported results have not been confirmed by the subsequent experiments. However, even if a few of the reported signals survive the test of time the underlying theory/theories must have multiple DM particles with masses in the range of a few keV to a few $\mathrm{TeV}$. The pMSSM with a single DM candidate can then at best be a part of a bigger scenario having multiple DM candidates. We, therefore, do not include these constraints in our analysis. 


\subsection{The simulation}

We follow the analysis by ATLAS Collaboration for Run I data where they introduced 20 signal regions (SR) each characterized by a set of cuts [42]. ${ }^{6}$ In tables 7 and 8 of [42] the model independent upper limit on $N_{B S M}$ at $95 \%$ CL for each SR is shown. A point in the parameter space of any model is said to be excluded if the corresponding $N_{B S M}$ exceeds the upper bound for atleast one of the $20 \mathrm{SRs}$ in [42]. We validate our simulation ([44] and [98]) and draw the exclusion contours for the models under consideration using PYTHIA. However, in this work we draw the exclusion contours considering the productions of all combinations of EWeakinos - heavy as well as light. For computing the NLO cross-sections of EWeakino production we use PROSPINO 2.1 [99].

We also simulate multilepton signals $(\geq 3 l)$ at $13 \mathrm{TeV}$ LHC using PYTHIA which is described in later sections. The judicious choice of cuts are made in order to tame down the potentially dangerous background in each case. The relevant background events are generated using ALPGEN (v 2.1) [100] with MLM matching [101, 102] and then the generated events are fed to PYTHIA for showering and hadronization. Reconstruction of jets is made following the anti- $k_{t}$ algorithm [103] by interfacing PYTHIA with FASTJET [104] with $R=0.4$. The reconstructed jets are required to have $P_{T}>20 \mathrm{GeV}$ and $|\eta|<2.5$. Also all the leptons (e and $\mu$ ) in the final state must have $P_{T}>10 \mathrm{GeV}$ and $|\eta|<2.5$. In addition to that, each of them is required to pass isolation cuts as defined by the ATLAS/CMS collaboration [42]-[43]. We use these selection cuts for all our analyses in this work. We use CTEQ6L [105] parton density function (PDF) in our simulations of all signals.

\subsection{Scanning the parameter space}

We use the following pMSSM parameters throughout this study. The squark masses belonging to the first two generations, $M_{A}$ and $M_{3}$ are set at a large value of $2 \mathrm{TeV} .{ }^{7}$ Note that these parameters do not have any effect on the EW sector which is our main concern in this paper. The trilinear coupling $A_{t}$ is set at $-2 \mathrm{TeV}$ so that the Higgs mass is consistent with the measured value. All other trilinear couplings namely $A_{b}, A_{\tau}, A_{u}, A_{d}, A_{e}$ are assumed to be zero. $M_{1}, M_{2}$ are scanned in the interval (see section 2) while $\tan \beta$ is fixed at a high value 30 . The parameter $\mu$ is chosen to ensure the characteristics of each model. Thus in the compressed model we take $\mu=1.05 M_{1} .{ }^{8}$ The choices for the other models will be given in the next section. The slepton masses are fixed as discussed in section 2. The $\mathrm{SM}$ parameters considered are $m_{t}^{\text {pole }}=175 \mathrm{GeV}, m_{b}^{\bar{M} S}=4.25 \mathrm{GeV}, m_{\tau}=1.77 \mathrm{GeV}$ and $M_{Z}=91.18 \mathrm{GeV}$. We consider only positive sign of $\mu$ in our work.

In this work we use Suspect(v 2.41) [106] for obtaining mass spectra and for evaluating $a_{\mu}^{S U S Y}$. We compute the decay BRs of sparticles using SUSYHIT [107]. Calculations of relic density and $\sigma^{S I}$ are done with micrOMEGAs (v3.2) [108].

\footnotetext{
${ }^{6}$ It may be noted that ref. [51] was based on earlier RUN-I data [97].

${ }^{7}$ Some of the large masses (e.g. $m_{\tilde{g}}$ or $m_{A}$ ) introduces a modest degree of fine tuning.

${ }^{8}$ The consequences for other choices will be discussed in the next section.
} 


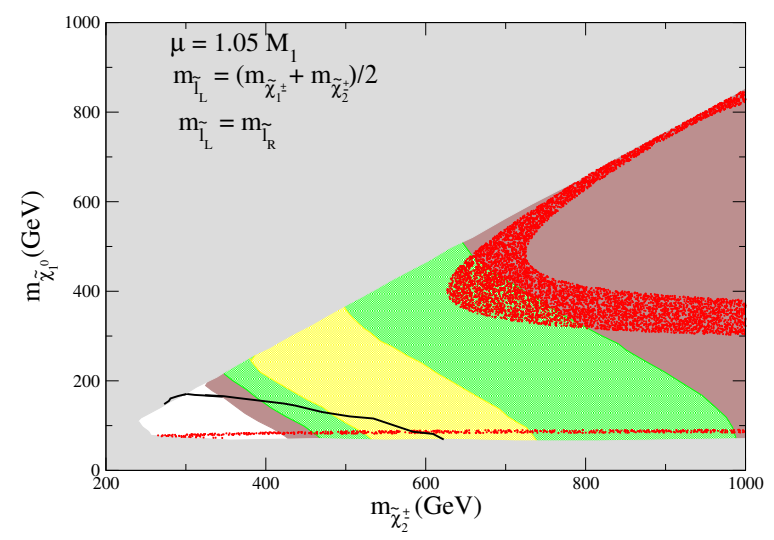

Figure 1. The black line represents the exclusion contour in $m_{\tilde{\chi}_{2}^{ \pm}}-m_{\tilde{\chi}_{1}^{0}}$ plane in the compressed model obtained by our simulation using ATLAS data [42]. The brown, green and yellow regions in the parameter space are consistent with $a_{\mu}$ data at $3 \sigma, 2 \sigma$ and $1 \sigma$ level respectively. The red points satisfy WMAP/PLANCK data of DM relic density.

\section{Constraining models with non-decoupled heavier EWeakinos}

In this section we delineate the APS of the models described in section 2 using the constraints discussed in subsection 4.1.

\subsection{Compressed LHHS model}

As already discussed the compressed LHHS or simply the compressed Model model with $\mu \approx M_{1}$ and approximately mass degenerate LSP and other lighter EWeakinos is attractive from the point of view of DM relic density and naturalness $([9,36-39,54])$. On the other hand the heavier EWeakinos - if non-decoupled - can lead to viable multilepton signals at the LHC as we shall show in this and the next section.

In figure 1 we display the constraints in the $m_{\widetilde{\chi}_{1}^{0}}-m_{\widetilde{\chi}_{2}^{ \pm}}$plane of the compressed model (see section 2.1). The black exclusion contour, which shows the constraint on $m_{\widetilde{\chi}_{2}^{ \pm}}$[52], from the ATLAS $3 l+\mathbb{E}_{T}$ data. For $m_{\widetilde{\chi}_{1}^{0}} \simeq 80 \mathrm{GeV}$, there is a strong bound $m_{\widetilde{\chi}_{2}^{ \pm}}>610 \mathrm{GeV}$. The LSP mass can not be lowered further due to the LEP bound $m_{\tilde{\chi}_{1}^{ \pm}}>103 \mathrm{GeV}$ [109]. However, note that for $m_{\widetilde{\chi}_{1}^{0}} \geq 170 \mathrm{GeV}$, there is no bound on $m_{\widetilde{\chi}_{2}^{ \pm}}$. On the whole it follows that the sensitivity to the $3 l$ data increases significantly if the heavier EWeakinos are nondecoupled. The exclusion is weaker for $m_{\widetilde{\chi}_{2}^{ \pm}}<300 \mathrm{GeV}$, since the higgsino component in $\widetilde{\chi}_{2}^{ \pm}$dominantes over the wino component. As a result the production cross-section is suppressed.

The dominant contribution to $a_{\mu}$ comes from chargino - sneutrino loop. Although the loop involving $\widetilde{\chi}_{1}^{ \pm}$dominates, the loop contribution of $\widetilde{\chi}_{2}^{ \pm}$(which is almost half of the former) comes with an opposite sign. Their combined effect helps to get the $a_{\mu}$ value in the right ballpark. Different coloured regions in figure 1 (see the figure caption) represent different levels of agreement between the model predictions and the data. This also indicates that over a fairly large parameter space better agreement than the SM is obtained. 


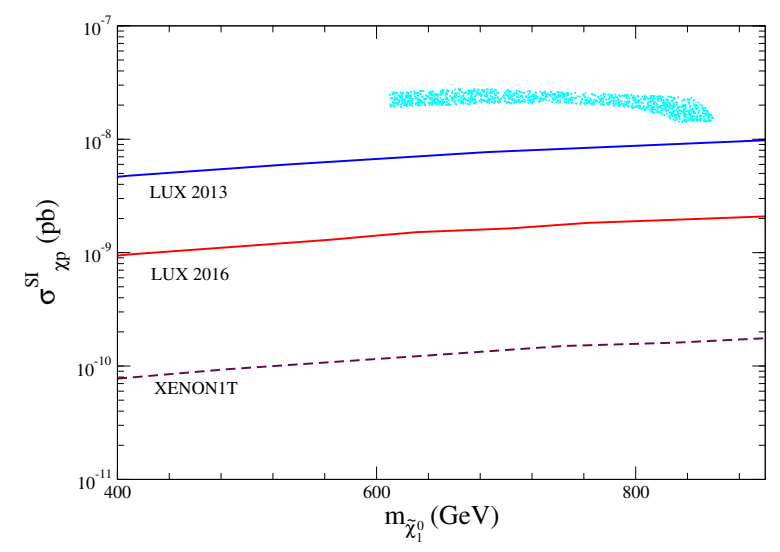

Figure 2. Direct detection results for compressed model. LUX and XENON1T results are shown as solid red and dashed magenta lines respectively. Blue points satisfy WMAP/PLANCK data, LHC constraints and $a_{\mu}$ up to the level of $2 \sigma$.

There are several distinct regions consistent with WMAP/PLANCK constraints in figure 1. The main DM producing mechanism for the upper branch of the parabola like region is LSP $-\widetilde{\chi}_{1}^{ \pm}$coannihilation. Some contributions also come from LSP pair annihilations into $W^{+} W^{-}$mediated by $\widetilde{\chi}_{1}^{ \pm}$and $\widetilde{\chi}_{2}^{ \pm}$although they are subdominant due to relatively large $m_{\tilde{\chi}_{1}^{ \pm}}$. The lower branch of the parabola arises mainly due to $\tilde{\chi}_{1}^{ \pm}$mediated LSP pair annihilations into $W^{+} W^{-}$, into $Z Z$ through $\widetilde{\chi}_{2}^{0}$ or $\widetilde{\chi}_{3}^{0}$ and $t \bar{t}$ through virtual $Z$ exchange. Here LSP $-\widetilde{\chi}_{1}^{ \pm}$coannnihilation is small. In the lowest band at fixed $m_{\widetilde{\chi}_{1}^{0}}$, LSP pair annihilations into $W^{+} W^{-}$via $\widetilde{\chi}_{1}^{ \pm}$is the main process. However, annihilation into $f \bar{f}$ final states has a non negligible contribution. Note that, there is no region where DM production proceeds via $h$ or $Z$ resonance since the LSP masses required for these processes are not allowed by the mass bound on $\widetilde{\chi}_{1}^{ \pm}$from LEP.

It may be noted that in the parabola like region $m_{\tilde{\chi}_{2}^{ \pm}}$is rather high $(>600 \mathrm{GeV})$ while in the lower band relatively small values of $m_{\widetilde{\chi}_{2}^{ \pm}}$are ruled out by the LHC constraints. Such high values of $m_{\tilde{\chi}_{2}^{ \pm}}$tend to suppress the multilepton signals below the observable level for integrated luminosities expected to accumulate before the next long shutdown (see the next section).

The DM relic density constraint is compatible with lighter $\widetilde{\chi}_{2}^{ \pm}$if extreme compression, represented by the choice $\mu=1.05 M_{1}$ is relaxed to some extent. For extreme compression the mass gap between LSP and $\widetilde{\chi}_{1}^{ \pm}$is approximately $30 \mathrm{GeV}$ irrespective of the specific choice of $M_{1}$. As a result both LSP - LSP annihilation and LSP $-\tilde{\chi}_{1}^{ \pm}$co-annihilation are pronounced yielding the observed DM relic density. For $m_{\tilde{\chi}_{2}^{ \pm}}<600 \mathrm{GeV}$ the wino component in LSP is larger which further enhances the LSP annihilation rate leading to under abundance of DM relic density. If the compression is relaxed to some extent by increasing the ratio $\mu / M_{1}$ the WMAP/PLANCK constraint can be satisfied for lower $m_{\widetilde{\chi}_{2}^{ \pm}}$ favourable for multilepton signals. We will illustrate the impact of the modified scenario on multilepton signals in the next section with the help of BPs. 


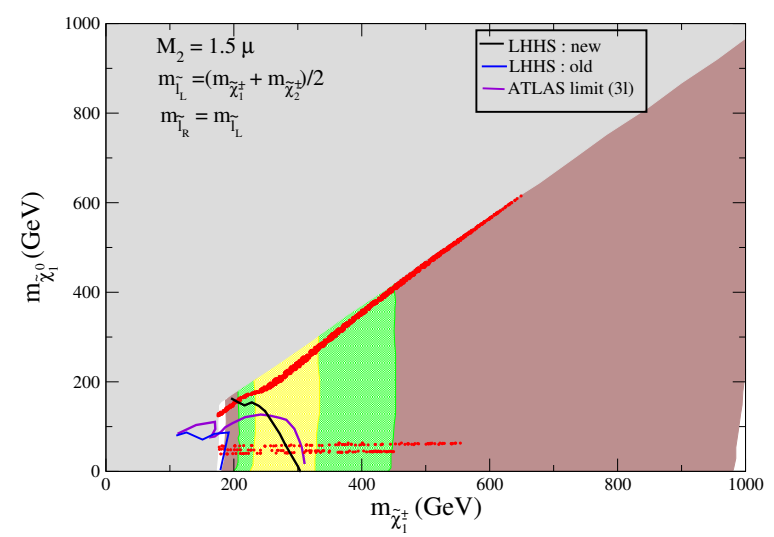

Figure 3. Plot in $m_{\widetilde{\chi}_{1}^{ \pm}}-m_{\tilde{\chi}_{1}^{0}}$ plane in Light Higgsino and Heavy Slepton (LHHS) model. The black contour is for the non-decoupled scenario whereas the blue one is for the decoupled scenario (see text for details). The magenta line is the exclusion contour as obtained by ATLAS in case of LWHS Model. Colors and conventions are same as in figure 1.

It has already been noted that the recent LUX upper bounds on $\sigma^{S I}$ [91] are in conflict with bino-higgsino DM [110] with low masses. We plot in figure 2 the prediction for $\sigma^{S I}$ in the compressed model for the APS in figure 1. It follows that $\sigma^{S I}$ is typically $\simeq 10^{-8}$ $\mathrm{pb}$ for $\widetilde{\chi}_{1}^{0} \simeq 600 \mathrm{GeV}$ whereas the LUX upper bound on $\sigma_{S I}$ is smaller by a factor of 15 for this LSP mass. However, the theoretical prediction involves several uncertainties (see section 4.1) including the critical one - an uncertain value of $\rho_{E}$. The possibility that the predicted values are significantly weaker are, therefore, wide open. We, therefore, do not wish to exclude any model at this stage on the basis of the direct detection data.

\subsection{LHHS model}

In figure 3 we show our result in LHHS model (see section 2.2). The black line represents the exclusion contour in the non-decoupled scenario at LHC RUN I whereas the blue line represents a much weaker exclusion contour in the decoupled scenario [45]. The magenta line represents the ATLAS exclusion in the LWHS model - the strongest limit derived in the $m_{\widetilde{\chi}_{1}^{0}}-m_{\widetilde{\chi}_{1}^{ \pm}}$plane from RUN I data. As can be seen from the plot, the constraints are significantly stronger due to the presence of heavier EWeakinos. For negligible LSP mass, the bound on $m_{\widetilde{\chi}_{1}^{ \pm}}$is found to be $\gtrsim 300 \mathrm{GeV}$ (earlier it was $\gtrsim 175 \mathrm{GeV}$ [45]). On the other hand, for LSP mass $\gtrsim 165 \mathrm{GeV}$, there is no bound on $m_{\widetilde{\chi}_{1}^{ \pm}}$. In the decoupled scenario, the corresponding result was $m_{\widetilde{\chi}_{1}^{ \pm}} \geq 100 \mathrm{GeV}$. The dominant contribution to $a_{\mu}$ comes from chargino - sneutrino loop.

There are two separate branches consistent with the WMAP/PLANCK constraints. The dominant contributions to DM relic density in the upper branch comes from LSP pair annihilation into $W^{+} W^{-}$mediated by $\widetilde{\chi}_{1}^{ \pm}$, annihilation into $Z Z$ and $t \bar{t}$ through virtual $Z$ exchanges. There is also some contributions from LSP $-\tilde{\chi}_{1}^{ \pm}$coannihilation. In the lower branch, the main mechanism is LSP annihilation mediated by $Z$ and $h$ resonances.

The recent stringent constraints provided by the LUX experiment $([91])$, however, question the viability of this model. However, the uncertainties in the prediction of $\sigma_{S I}$ limits 


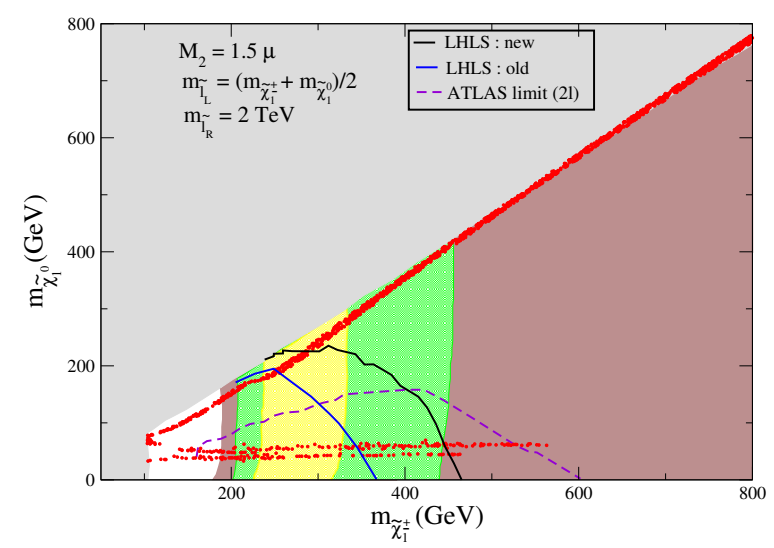

Figure 4. Plot in $m_{\widetilde{\chi}_{1}^{ \pm}}-m_{\widetilde{\chi}_{1}^{0}}$ plane in Light Higgsino and light Left Slepton (LHLS) model. The black contour is for the non-decoupled scenario whereas the blue one is for the decoupled scenario (see text for details). The purple dashed line represents the exclusion contour from ATLAS slepton searches [60]. Colors and conventions are same as in figure 1.

extracted from the data as discussed in the last section 4.1 does not allow a definite conclusion. For LSP masses of a few hundred $\mathrm{GeV}$ the upper limit on $\sigma^{S I}$ is typically of the order of a few $\times 10^{-1} \mathrm{zb}$ (i.e. $10^{-10} \mathrm{pb}$ ). On the other hand a glance at figure 8 of [45] indicate that in this model the prediction is $\sim 10 \mathrm{zb}$ in most cases. However, for LSP masses corresponding to the $\mathrm{Z}$ or $\mathrm{H}$ mediated resonant production of DM relic density, $\sigma^{S I}$ is much lower and is still consistent with the stringent LUX data.

\subsection{LHLS model}

Figure 4 represents our result in LHLS model (see section 2.3). The colour conventions for the contours are same as those in previous section. The exclusion contour in the nondecoupled scenario is significantly stronger than the corresponding decoupled scenario. If the LSP mass is negligible, $\widetilde{\chi}_{1}^{ \pm}$masses approximately upto $460 \mathrm{GeV}$ are excluded from LHC trilepton search. Note that, in decoupled scenario this limit was considerably weaker $\sim$ $365 \mathrm{GeV}$. Also, for LSP mass $\gtrsim 230 \mathrm{GeV}$ (which was $\gtrsim 200 \mathrm{GeV}$ in the decoupled scenario), there is no bound on $\widetilde{\chi}_{1}^{ \pm}$mass. As in the previous case, chargino-sneutrino loop dominates in case of $a_{\mu}$.

$\widetilde{\chi}_{1}^{ \pm}$mediated LSP pair annihilation into $W^{+} W^{-}$and annihilation into $t \bar{t}$ through virtual $Z$ exchange are the main contributing processes in the upper branch consistent with WMAP/PLANCK constraint. Small amount of annihilations into $Z Z$ and $Z h$ are also present. As $m_{\tilde{\chi}_{1}^{ \pm}}$increases, $W^{+} W^{-}$and $t \bar{t}$ productions become subdominant and $\tilde{\chi}_{1}^{ \pm}$ coannihilation takes over. Small amount of $\widetilde{\chi}_{2}^{0}$ coannihilation is also present. A large part of this upper branch at high $m_{\widetilde{\chi}_{1}^{ \pm}}$is disfavoured by the $a_{\mu}$ constraint. In the lower branch, $Z$ and $h$ production processes give the DM relic density in the right ballpark. The lower branch is strongly disfavoured by the LHC data or by the $a_{\mu}$ constraint. From figure $7 \mathrm{a}$ of [45] it follows that $\sigma^{S I}$ corresponding to the APS of this model violates the recent LUX bound by factors of $7-8$. 


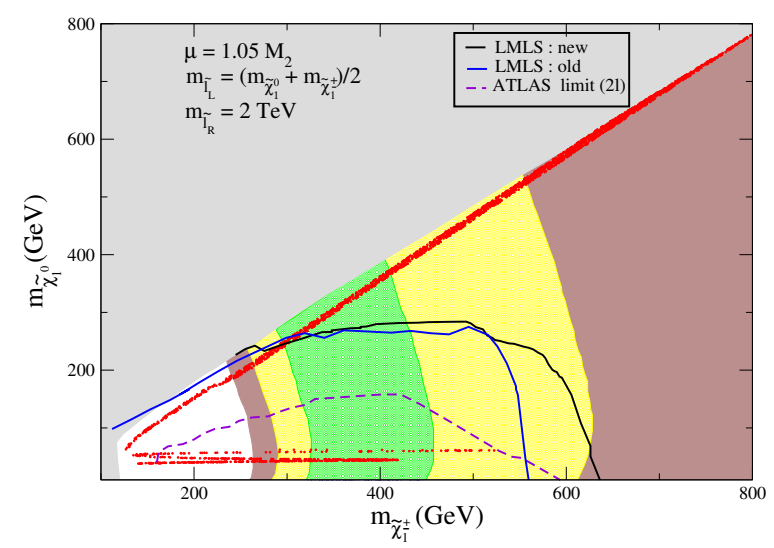

Figure 5. Plot in $m_{\widetilde{\chi}_{1}^{ \pm}}-m_{\widetilde{\chi}_{1}^{0}}$ plane in Light Mixed and light Left Slepton (LMLS) model. The black contour is for the non-decoupled scenario whereas the blue one is for the decoupled scenario (see text for details). Magenta dashed line represents the exclusion contour from ATLAS slepton searches. Colors and conventions are same as in figure 1.

\subsection{LMLS model}

The APS for the LMLS model (see section 2.4) is shown in figure 5. $\widetilde{\chi}_{1}^{ \pm}$masses approximately upto $630 \mathrm{GeV}$ are excluded for a massless LSP whereas LHC slepton searches put a bound $\sim 600 \mathrm{GeV}$ on $m_{\tilde{\chi}_{1}^{ \pm}}$for vanishing small LSP mass. The exclusion limit is considerably stronger than the decoupled scenario. Major contribution to $a_{\mu}$ is provided by chargino-sneutrino loop.

The WMAP/PLANCK data are satisfied by the points in the upper branch. Here DM production is mainly contributed by annihilations into $W^{+} W^{-}$and $t \bar{t}$ pairs. Small amount of annihilation into $Z Z$ is also present. Also non-negligible contributions come from LSPsneutrino and LSP-slepton coannihilations. In the lower branch, $Z$ and $h$ resonance produce the correct DM relic density. In this case also, the lower branch is completely ruled out by the LHC data. A small part of the total parameter space is available which is consistent with all the constraints. The conflict between $\sigma^{S I}$ and the LUX bound persists in this model.

\section{The multilepton signatures}

In this section, we focus on the prospect of discovering several multilepton signatures in models discussed in section $2 .{ }^{9}$ We shall show below that it may be possible to discriminate against the models by the relative rates of signals in different channels. We present our results for an integrated luminosity of $100 \mathrm{fb}^{-1}$ which is expected to accumulate before the next long shutdown of the LHC. We do not consider the LMLS model since in this case the $m l+\mathbb{E}_{T}$ signatures for $m>3$ do not look very promising for the above integrated luminosity. We consider 1) $3 l, 2) 4 l, 3)$ SS3OS1l (three same sign and one opposite sign leptons) and 4) $5 l$ final states all accompanied by large $\mathbb{E}_{T}$ coming from all possible EWeakino pairs - the lighter as well as the heavier ones. It may be noted that the last two signals were

\footnotetext{
${ }^{9}$ Multilepton signatures with decoupled sleptons were studied in [53].
} 


\begin{tabular}{|c|c|c|c|c|c|c|c|c|}
\hline \multirow{2}{*}{$\begin{array}{c}\text { Parameters/ } \\
\text { Masses }\end{array}$} & \multicolumn{4}{|c|}{ Compressed } & \multicolumn{5}{c|}{ LHHS } \\
\cline { 2 - 9 } & BP1 & BP2 & BP3 & BP4 & BP1 & BP2 & BP3 & BP4 \\
\hline$M_{1}$ & 213 & 185 & 144 & 134 & 176 & 165 & 74 & 32 \\
\hline$\mu$ & 223.6 & 194.2 & 145.9 & 140.7 & 240 & 285 & 317 & 381 \\
\hline$M_{2}$ & 360 & 466 & 594 & 667 & 360 & 427.5 & 475.5 & 571.5 \\
\hline$m_{\widetilde{\chi}_{1}^{0}}$ & 180 & 155 & 113 & 106 & 162 & 157 & 71 & 31 \\
\hline$m_{\widetilde{\chi}_{1}^{ \pm}}$ & 213 & 191 & 150 & 142 & 228 & 275 & 308 & 375 \\
\hline$m_{\widetilde{\chi}_{2}^{ \pm}}$ & 401 & 500 & 628 & 700 & 403 & 469 & 516 & 612 \\
\hline
\end{tabular}

Table 4. BPs consistent with the LHC and $a_{\mu}$ constraints as derived in section 5. All masses and mass parameters are in $\mathrm{GeV}$.

first studied in [52]. Here we evaluate the discovery potential of the signals after taking into account the constraints derived in the last section. The observation that the heavier EWeakinos are crucial for observing final states with more than 3 leptons (see section 3 ) will be confirmed by generator level simulation of the signals and the corresponding backgrounds for selected BPs. In our analysis we make the simplistic assumption that $S / \sqrt{B} \gtrsim 5$ is sufficient to claim a discovery. In case the background is negligible 5 signal events are taken as the criterion for discovery. Some of the leptonic channels have been extensively studied during RUN I of the LHC in models with decoupled heavier EWeakinos [42, 43, 112]. In contrast our emphasis in this paper is on the non-decoupled scenarios following [52].

For each model, we have divided the BPs chosen for studying the multilepton signals into two categories:

- SET-I: BPs satisfying only the $3 l+\mathbb{E}_{T}$ and $a_{\mu}$ constraints.

- SET-II: BPs also satisfying the WMAP/PLANCK constraints.

SET-I takes into account the possibility that there may be a non-SUSY explanation of the observed DM relic density. The BPs are enlisted in tables 4 and table 5. All BPs are consistent with the new bounds derived in the last section.

In table 6 the BPs are taken from both the bands satisfying the DM relic density constraints (see figures 1 and 3). In the LHLS model, the lower band is excluded by the LHC and/or the $a_{\mu}$ constraints. In table 7 , we choose points only from the upper band.

\subsection{The prospective $3 l+\mathbb{E}_{T}$ signal before the next long shut down of the LHC}

The dominant SM backgrounds in this case are:

- $W Z$ production followed by leptonic decays of both $W$ and $Z$.

- $Z Z$ production with $Z$ decaying into leptons where one lepton goes missing.

- $t \bar{t} Z$ production followed by $Z \rightarrow l^{+} l^{-}, t(\bar{t}) \rightarrow b(\bar{b}) W$ and one of the $W$ bosons decays into leptons. 


\begin{tabular}{|c|c|c|c|}
\hline Parameters & \multicolumn{3}{|c|}{ LHLS } \\
\cline { 2 - 4 } Masses & BP1 & BP2 & BP3 \\
\hline$M_{1}$ & 275 & 241 & 204 \\
\hline$\mu$ & 290 & 347 & 405 \\
\hline$M_{2}$ & 435 & 520.5 & 607.5 \\
\hline$m_{\widetilde{\chi}_{1}^{0}}$ & 244 & 230 & 198 \\
\hline$m_{\widetilde{\chi}_{1}^{ \pm}}$ & 280 & 340 & 400 \\
\hline$m_{\widetilde{\chi}_{2}^{ \pm}}$ & 475 & 560 & 646 \\
\hline
\end{tabular}

Table 5. BPs consistent with the LHC and $a_{\mu}$ constraints as derived in section 5. All masses and mass parameters are in $\mathrm{GeV}$.

\begin{tabular}{|c|c|c|c|c|c|c|c|c|}
\hline \multirow{2}{*}{$\begin{array}{c}\text { Parameters } \\
\text { Masses }\end{array}$} & \multicolumn{4}{|c|}{ Compressed } & \multicolumn{4}{c|}{ LHHS } \\
\cline { 2 - 9 } & BP1-DM & BP2-DM & BP3-DM & BP4-DM & BP1-DM & BP2-DM & BP3-DM & BP4-DM \\
\hline$M_{1}$ & 380 & 382 & 116 & 116 & 200 & 276 & 70 & 60 \\
\hline$\mu$ & 399 & 401.1 & 121.8 & 121.8 & 266 & 325 & 349 & 405 \\
\hline$M_{2}$ & 609 & 662 & 666 & 736 & 399 & 487.5 & 523.5 & 607.5 \\
\hline$m_{\widetilde{\chi}_{1}^{0}}$ & 350 & 356 & 87 & 88 & 186 & 258 & 70 & 60 \\
\hline$m_{\tilde{\chi}_{1}^{ \pm}}$ & 393 & 400 & 123 & 123 & 255 & 317 & 342 & 400 \\
\hline$m_{\widetilde{\chi}_{2}^{ \pm}}$ & 650 & 700 & 700 & 770 & 440 & 528 & 564 & 647 \\
\hline
\end{tabular}

Table 6. BPs consistent with the LHC, $a_{\mu}$ and WMAP/PLANCK constraints as derived in section 5. All masses and mass parameters are in $\mathrm{GeV}$.

\begin{tabular}{|c|c|c|}
\hline \multirow{2}{*}{$\begin{array}{c}\text { Parameters } \\
\text { Masses }\end{array}$} & \multicolumn{2}{|c|}{ LHLS } \\
\cline { 2 - 3 } & BP1-DM & BP2-DM \\
\hline$M_{1}$ & 277 & 403 \\
\hline$\mu$ & 328 & 425 \\
\hline$M_{2}$ & 492 & 637.5 \\
\hline$m_{\widetilde{\chi}_{1}^{0}}$ & 258 & 376 \\
\hline$m_{\widetilde{\chi}_{1}^{ \pm}}$ & 320 & 420 \\
\hline$m_{\widetilde{\chi}_{2}^{ \pm}}$ & 530 & 676 \\
\hline
\end{tabular}

Table 7. BPs consistent with the LHC, $a_{\mu}$ and DM relic density constraints as derived in section 5 . All masses and mass parameters are in $\mathrm{GeV}$. 


\begin{tabular}{|c|c|c|c|c|c|}
\hline Background & $\sigma_{\text {prod }}$ & \multicolumn{5}{|c|}{$\sigma_{\text {eff }}^{3 l}$ in $f b$} \\
\cline { 3 - 6 } Processes & in $p b$ & after & after & after & after \\
& & $A 1$ & $A 2$ & $A 3$ & $A 4$ \\
\hline$W Z$ & 32.69 & 168.3 & 13.11 & 0.18 & 0.17 \\
$Z Z$ & 10.63 & 16.5 & 1.25 & 0.007 & 0.006 \\
$t \bar{t} Z$ & 0.018 & 1.95 & 0.39 & 0.015 & 0.010 \\
$W W Z$ & 0.133 & 1.33 & 0.17 & 0.013 & 0.012 \\
$W Z Z$ & 0.042 & 0.54 & 0.044 & 0.005 & 0.004 \\
$Z Z Z$ & 0.010 & 0.05 & 0.003 & 0.0001 & 0.0001 \\
$W W W$ & 0.159 & 0.79 & 0.07 & 0.059 & 0.059 \\
\hline Total & & & & & \\
background & 43.68 & & & & 0.261 \\
\hline
\end{tabular}

Table 8. The production and effective cross-sections $\left(\sigma_{\text {eff }}^{3 l}\right)$ after all cuts of different SM backgrounds.

- $V V V$ where $(V=W, Z)$ production where leptonic decays of $W$ and $Z$ lead to trileptonic final states.

The following sets of cuts have been used in our analysis to suppress the backgrounds:

- A1) Events with exactly 3 isolated leptons passing the selection cuts mentioned in section 4.2 are required.

- A2) Events with invariant mass of any Same Flavour Opposite Sign (SFOS) lepton pair falling within the window $81.2 \mathrm{GeV}<m_{\text {inv }}<101.2 \mathrm{GeV}$ are vetoed out.

- A3) Events are required to have atleast $\mathbb{E}_{T}>200 \mathrm{GeV}$.

- A4) Finally b-veto [111] is applied to reduce the potentially strong background from $t \bar{t} Z$.

We present the estimated number of background and signal events for an integrated luminosity of $100 \mathrm{fb}^{-1}$ in tables $8-10$. For the compressed model, all the BPs corresponding to SET-I can lead to discovery for the quoted luminosity. However, for SET-II the $3 l$ signal is rather weak except for BP3-DM. The others may be relevant as higher luminosities accumulate after the next long shutdown. For LHHS and LHLS Model, both sets of BPs (SET-I and SET-II) give observable $3 l$ signal.

\subsection{The prospective $4 l+\boldsymbol{E}_{T}$ signal}

In this section we assess the discovery potential of the $4 l$ channel at LHC RUN II. It may be noted that our analysis based on the pMSSM is more general than the ATLAS 41 analysis [112] in a specific simplified model. However, we have checked that the models 


\begin{tabular}{|c|c|c|c|c|c|c|c|c|}
\hline \multirow[b]{2}{*}{ Models } & \multirow{2}{*}{$\begin{array}{c}\text { Benchmark } \\
\text { Points }\end{array}$} & \multirow{2}{*}{$\begin{array}{l}\sigma_{\text {prod }} \\
\text { in } f b\end{array}$} & \multicolumn{4}{|c|}{$\sigma_{\text {eff }}^{3 l}$ in $f b$} & \multirow[b]{2}{*}{$\begin{array}{c}\text { Total } 3 l \\
\text { events }\end{array}$} & \multirow[b]{2}{*}{$S / \sqrt{B}$} \\
\hline & & & $\begin{array}{c}\text { after } \\
A 1\end{array}$ & $\begin{array}{c}\text { after } \\
A 2\end{array}$ & $\begin{array}{c}\text { after } \\
A 3\end{array}$ & $\begin{array}{c}\text { after } \\
A 4\end{array}$ & & \\
\hline \multirow{8}{*}{ Compressed } & $\mathrm{BP} 1$ & 507.9 & 7.04 & 5.89 & 0.52 & 0.48 & 48.2 & 9.1 \\
\hline & BP2 & 650.2 & 4.28 & 3.82 & 0.58 & 0.54 & 54.6 & 10.3 \\
\hline & BP3 & 1417. & 4.84 & 4.59 & 0.60 & 0.58 & 58.1 & 10.9 \\
\hline & $\mathrm{BP} 4$ & 1763. & 4.05 & 4.01 & 0.27 & 0.26 & 26.4 & 4.9 \\
\hline & BP1-DM & 56.38 & 0.67 & 0.57 & 0.13 & 0.12 & 12.1 & 2.3 \\
\hline & BP2-DM & 51.21 & 0.49 & 0.42 & 0.12 & 0.10 & 10.8 & 2.0 \\
\hline & BP3-DM & 2817. & 5.52 & 5.27 & 0.34 & 0.33 & 33.8 & 6.4 \\
\hline & BP4-DM & 2812. & 6.10 & 6.04 & 0.20 & 0.16 & 16.8 & 3.2 \\
\hline \multirow{8}{*}{ LHHS } & $\mathrm{BP} 1$ & 555.4 & 6.80 & 0.78 & 0.67 & 0.66 & 66.6 & 12.6 \\
\hline & $\mathrm{BP} 2$ & 301.4 & 4.53 & 0.74 & 0.49 & 0.49 & 49.1 & 9.3 \\
\hline & BP3 & 200.5 & 2.62 & 0.97 & 0.57 & 0.57 & 57.5 & 10.8 \\
\hline & $\mathrm{BP} 4$ & 92.56 & 1.16 & 0.59 & 0.30 & 0.30 & 30.8 & 5.8 \\
\hline & BP1-DM & 370.8 & 4.34 & 0.48 & 0.35 & 0.35 & 35.2 & 6.7 \\
\hline & BP2-DM & 156.3 & 1.84 & 0.29 & 0.22 & 0.22 & 22.0 & 4.2 \\
\hline & BP3-DM & 134.8 & 1.64 & 0.79 & 0.46 & 0.46 & 46.9 & 8.9 \\
\hline & BP4-DM & 71.12 & 0.84 & 0.45 & 0.25 & 0.25 & 25.4 & 4.8 \\
\hline
\end{tabular}

Table 9. The production cross-sections of all EWeakino pairs and $\sigma_{\text {eff }}^{3 l}$ for the BPs defined in table 4 and table 6 . Also the total number of $3 l$ events along with $S / \sqrt{B}$ are shown for an integrated luminosity of $100 \mathrm{fb}^{-1}$.

\begin{tabular}{|c|c|c|c|c|c|c|c|c|}
\hline \multirow[b]{2}{*}{ Models } & \multirow{2}{*}{$\begin{array}{c}\text { Benchmark } \\
\text { Points }\end{array}$} & \multirow{2}{*}{$\begin{array}{l}\sigma_{\text {prod }} \\
\text { in } f b\end{array}$} & \multicolumn{4}{|c|}{$\sigma_{\mathrm{eff}}^{3 l}$ in $f b$} & \multirow[b]{2}{*}{$\begin{array}{c}\text { Total } 3 l \\
\text { events }\end{array}$} & \multirow[b]{2}{*}{$S / \sqrt{B}$} \\
\hline & & & $\begin{array}{c}\text { after } \\
A 1\end{array}$ & $\begin{array}{c}\text { after } \\
A 2\end{array}$ & $\begin{array}{c}\text { after } \\
A 3\end{array}$ & $\begin{array}{c}\text { after } \\
A 4\end{array}$ & & \\
\hline \multirow{5}{*}{ LHLS } & $\mathrm{BP} 1$ & 210.1 & 9.9 & 9.1 & 0.96 & 0.92 & 92.0 & 17.4 \\
\hline & BP2 & 131.5 & 8.66 & 5.97 & 1.02 & 0.98 & 98.5 & 18.6 \\
\hline & BP3 & 69.93 & 3.02 & 2.41 & 0.82 & 0.78 & 78.3 & 14.8 \\
\hline & BP1-DM & 151.8 & 111.2 & 102.2 & 1.04 & 0.98 & 98.5 & 18.6 \\
\hline & BP2-DM & 45.25 & 2.26 & 2.09 & 0.35 & 0.32 & 32.5 & 6.15 \\
\hline
\end{tabular}

Table 10. The production cross-sections of all EWeakino pairs and $\sigma_{\text {eff }}^{3 l}$ for the BPs defined in table 5 and table 7 . Also the total number of $3 l$ events along with $S / \sqrt{B}$ are shown for an integrated luminosity of $100 \mathrm{fb}^{-1}$. 


\begin{tabular}{|c|c|c|c|c|c|}
\hline Background & \multirow{2}{*}{$\sigma_{\text {prod }}$} & \multicolumn{4}{|c|}{$\sigma_{\text {eff }}^{4 l}$ in $f b$} \\
\cline { 3 - 6 } Processes & in $p b$ & after & after & after & after \\
& & $B 1$ & $B 2$ & $B 3$ & $B 4$ \\
\hline$Z Z$ & 10.63 & 14.2 & 0.081 & 0 & 0 \\
$t \bar{t} Z$ & 0.018 & 0.26 & 0.039 & 0.018 & 0.005 \\
$W W Z$ & 0.133 & 0.18 & 0.012 & 0.004 & 0.002 \\
$W Z Z$ & 0.042 & 0.068 & 0.0014 & 0.0003 & 0.0003 \\
$Z Z Z$ & 0.010 & 0.04 & 0.0003 & 0.00005 & 0.00005 \\
\hline
\end{tabular}

Table 11. The production and effective cross-sections $\left(\sigma_{\text {eff }}^{4 l}\right)$ after the cuts for different SM backgrounds.

considered in this paper are not very sensitive to this data. The heavier EWeakinos play pivotal role in this case (see section 3). The main SM backgrounds are:

- $Z Z$ production where both $Z$ decay leptonically.

- $t \bar{t} Z$ production followed by $Z \rightarrow l^{+} l^{-}$and leptonic decays of the $W^{ \pm}$bosons coming from the top decay.

- $V V V$ with $Z$ and $W^{ \pm}$decaying into leptons.

The size of the SM background is considerably smaller than that for the trilepton final states. We apply the following set of cuts to make the background negligible:

- B1) Exactly 4 isolated leptons passing all the selection cuts (see section 4.2) are required.

- B2) The invariant mass of any SFOS lepton pair should not fall within the window $81.2-101.2 \mathrm{GeV}$.

- B3) Events must have $\mathbb{E}_{T}>80 \mathrm{GeV}$.

- B4) A b-veto is applied to suppress the background coming from $t \bar{t} Z$.

Tables 11-13 summarize the results. The total background is found to be vanishingly small after the cuts. For the compressed model, most of the BPs belonging to both the sets indicate potential discovery chances for $L=100 \mathrm{fb}^{-1}$. In case of LHHS model, BPs of SET-I can give rise to large $4 l+\mathbb{E}_{T}$ signal except the last one. For WMAP/PLANCK data satisfying points, the result is weaker for comparatively heavy $\widetilde{\chi}_{1}^{ \pm}$. Finally for LHLS model, the BPs of both sets lead to sufficiently large $4 l+\mathbb{E}_{T}$ signal.

\subsection{Three same sign and one opposite sign leptons (SS3OS1) $+\boldsymbol{E}_{T}$ signal}

We now discuss a special case of $4 l+\mathbb{E}_{T}$ signal when the total charge of the final state leptons is necessarily non-zero. This is of particular interest as the corresponding SM 


\begin{tabular}{|c|c|c|c|c|c|c|c|}
\hline \multirow[b]{2}{*}{ Models } & \multirow{2}{*}{$\begin{array}{c}\text { Benchmark } \\
\text { Points }\end{array}$} & \multirow{2}{*}{$\begin{array}{l}\sigma_{\text {prod }} \\
\text { in } f b\end{array}$} & \multicolumn{4}{|c|}{$\sigma_{\text {eff }}^{4 l}$ in $f b$} & \multirow[b]{2}{*}{$\begin{array}{l}\text { Total } 4 l \\
\text { events }\end{array}$} \\
\hline & & & $\begin{array}{c}\text { after } \\
B 1\end{array}$ & $\begin{array}{c}\text { after } \\
B 2\end{array}$ & $\begin{array}{c}\text { after } \\
B 3\end{array}$ & $\begin{array}{c}\text { after } \\
B 4\end{array}$ & \\
\hline \multirow{8}{*}{ Compressed } & BP1 & 507.9 & 1.18 & 0.78 & 0.48 & 0.46 & 46.7 \\
\hline & BP2 & 650.2 & 0.56 & 0.42 & 0.32 & 0.29 & 29.2 \\
\hline & BP3 & 1417. & 0.11 & 0.07 & 0.07 & 0.07 & 7.08 \\
\hline & BP4 & 1763. & 0.09 & 0.07 & 0.03 & 0.03 & 3.52 \\
\hline & BP1-DM & 56.38 & 0.10 & 0.06 & 0.05 & 0.04 & 4.62 \\
\hline & BP2-DM & 51.21 & 0.05 & 0.03 & 0.02 & 0.02 & 2.61 \\
\hline & BP3-DM & 2817. & 0.22 & 0.19 & 0.14 & 0.14 & 14.1 \\
\hline & BP4-DM & 2812. & 0.14 & 0.11 & 0.05 & 0.05 & 5.62 \\
\hline \multirow{8}{*}{ LHHS } & BP1 & 555.4 & 0.70 & 0.45 & 0.25 & 0.24 & 24.9 \\
\hline & $\mathrm{BP} 2$ & 301.4 & 0.41 & 0.32 & 0.10 & 0.10 & 10.2 \\
\hline & BP3 & 200.5 & 0.22 & 0.18 & 0.06 & 0.06 & 6.41 \\
\hline & $\mathrm{BP} 4$ & 92.56 & 0.08 & 0.07 & 0.03 & 0.02 & 2.86 \\
\hline & BP1-DM & 370.8 & 0.52 & 0.33 & 0.18 & 0.18 & 18.5 \\
\hline & BP2-DM & 156.3 & 0.19 & 0.15 & 0.10 & 0.10 & 10.1 \\
\hline & BP3-DM & 134.8 & 0.13 & 0.12 & 0.04 & 0.03 & 3.64 \\
\hline & BP4-DM & 71.12 & 0.07 & 0.07 & 0.02 & 0.02 & 2.34 \\
\hline
\end{tabular}

Table 12. The production cross-sections of all EWeakino pairs and $\sigma_{\text {eff }}^{4 l}$ for the BPs defined in table 4 and table 6 . Also the total number of $4 l$ events are shown for an integrated luminosity of $100 \mathrm{fb}^{-1}$.

\begin{tabular}{|c|c|c|c|c|c|c|c|}
\hline \multirow{4}{*}{ Models } & Benchmark & $\sigma_{\text {prod }}$ & \multicolumn{4}{|c|}{$\sigma_{\text {eff }}^{4 l}$ in $f b$} & \multirow{1}{*}{ Total $4 l$} \\
\cline { 5 - 7 } & Points & in $f b$ & after & after & after & after & Total \\
& & & $B 1$ & $B 2$ & $B 3$ & $B 4$ & events \\
\hline \multirow{5}{*}{ LHLS } & BP1 & 210.1 & 0.26 & 0.19 & 0.12 & 0.11 & 11.7 \\
& BP2 & 131.5 & 0.44 & 0.17 & 0.11 & 0.10 & 10.9 \\
& BP3 & 69.93 & 0.12 & 0.05 & 0.04 & 0.04 & 4.40 \\
\cline { 3 - 7 } & BP1-DM & 151.8 & 0.39 & 0.29 & 0.15 & 0.13 & 13.8 \\
& BP2-DM & 45.25 & 0.09 & 0.06 & 0.04 & 0.03 & 3.8 \\
\hline
\end{tabular}

Table 13. The production cross-sections of all EWeakino pairs and $\sigma_{\text {eff }}^{4 l}$ for the BPs defined in table 5 and table 7 . Also the total number of $4 l$ events are shown for an integrated luminosity of $100 f b^{-1}$. 


\begin{tabular}{|c|c|c|c|}
\hline \multirow{2}{*}{ Background } & \multirow{2}{*}{$\sigma_{\text {prod }}$} & \multicolumn{2}{|c|}{$\sigma_{\text {eff }}^{s s 30 s 1}$ in $f b$} \\
\cline { 3 - 4 } Processes & in $p b$ & after & after \\
& & $C 1$ & $C 2$ \\
\hline$t \bar{t} Z$ & 0.018 & 0.006 & 0.002 \\
$W Z Z$ & 0.042 & 0.007 & 0.003 \\
$Z Z Z$ & 0.010 & 0.0004 & 0.00003 \\
\hline
\end{tabular}

Table 14. The production and effective cross-sections $\left(\sigma_{\text {eff }}^{s s 3 o s 1}\right)$ after the cuts of different SM backgrounds.

background is very small and can be made to vanish by applying moderate cuts. Other interesting features will be discussed below. The main backgrounds are:

- $t \bar{t} Z$ production.

- $W Z Z$ production followed by leptonic decays of all the gauge bosons where one lepton fails to pass the selection cuts.

- $Z Z Z$ production.

Requiring 4 isolated leptons with non-zero total charge $(\mathrm{C} 1))$ and $\left.\mathbb{E}_{T}>80 \mathrm{GeV}(\mathrm{C} 2)\right)$ are found to be effective for reducing the background significantly. This is shown in table 14 .

In table 15-16, we show the number of signal events surviving the successive cuts.

The background is practically zero after one applies the above cuts. Tables $13-15$ show our results for SS3OS1l signals. For compressed model, it is possible to get more than 5 signal events for some of the BPs in both SET-I and SET-II. Although in LHHS (except BP1) and LHLS models the SS3OS1l $+\mathbb{E}_{T}$ signal events never reach 5 for the considered value of integrated luminosity, we would like to mention that some of them may as well serve as a hint. Thus the observation of this signal before the next long shut down of the LHC may reduce the number of competing models.

\section{$6.45 l+t_{T}$ signal}

Next we discuss the prospects of observing 5 isolated leptons associated with missing energy in the final state coming from EWeakino productions at RUN II of LHC. A few SM processes can give rise to the corresponding background. We enlist them below:

- $t \bar{t} Z$ production where both $Z$ and $W^{ \pm}$bosons (coming from top decays) decay into leptons and the remaining one comes from a heavy quark decay.

- $W Z Z$ production followed by leptonic decays of all the gauge bosons.

- $Z Z Z$ production with leptonic decay of all $Z$ bosons.

Demanding 5 isolated leptons in the final state (D1)) itself reduces the number of background events significantly. A moderate cut $\left.\mathbb{E}_{T}>80 \mathrm{GeV}(\mathrm{D} 2)\right)$ is then sufficient to 


\begin{tabular}{|c|c|c|c|c|c|}
\hline \multirow[b]{2}{*}{ Models } & \multirow{2}{*}{$\begin{array}{l}\text { Benchmark } \\
\text { Points }\end{array}$} & \multirow{2}{*}{$\begin{array}{l}\sigma_{\text {prod }} \\
\text { in } f b\end{array}$} & \multicolumn{2}{|c|}{$\sigma_{\text {eff }}^{s s 3 o s 1}$ in $f b$} & \multirow[b]{2}{*}{$\begin{array}{c}\text { Total SS3OS11 } \\
\text { events }\end{array}$} \\
\hline & & & $\begin{array}{c}\text { after } \\
D 1\end{array}$ & $\begin{array}{c}\text { after } \\
D 2\end{array}$ & \\
\hline \multirow{6}{*}{ Compressed } & $\mathrm{BP} 1$ & 507.9 & 0.29 & 0.20 & 20.3 \\
\hline & BP2 & 650.2 & 0.17 & 0.15 & 15.6 \\
\hline & BP3 & 1417. & 0.014 & 0.014 & 1.42 \\
\hline & $\mathrm{BP} 4$ & 1763. & 0.035 & 0.017 & 1.76 \\
\hline & BP1-DM & 56.38 & 0.033 & 0.025 & 2.53 \\
\hline & BP3-DM & 2817. & 0.084 & 0.056 & 5.63 \\
\hline \multirow{8}{*}{ LHHS } & BP1 & 555.4 & 0.11 & 0.083 & 8.33 \\
\hline & BP2 & 301.4 & 0.045 & 0.039 & 3.92 \\
\hline & BP3 & 200.5 & 0.028 & 0.020 & 2.01 \\
\hline & $\mathrm{BP} 4$ & 92.56 & 0.017 & 0.013 & 1.38 \\
\hline & BP1-DM & 370.8 & 0.056 & 0.033 & 3.34 \\
\hline & BP2-DM & 156.3 & 0.036 & 0.028 & 2.81 \\
\hline & BP3-DM & 134.8 & 0.017 & 0.016 & 1.62 \\
\hline & BP4-DM & 71.12 & 0.011 & 0.010 & 1.06 \\
\hline
\end{tabular}

Table 15. The production cross sections of all EWeakino pairs and $\sigma_{\text {eff }}^{s s 3 o s 1}$ after successive cuts for the BPs defined in table 5 and table 7. Also the total number of SS3OS1l events are shown for an integrated luminosity of $100 \mathrm{fb}^{-1}$.

\begin{tabular}{|c|c|c|c|c|c|}
\hline \multirow{3}{*}{ Models } & Benchmark & $\sigma_{\text {prod }}$ & \multicolumn{2}{|c|}{$\sigma_{\text {eff }}^{s s 3 o s}$ in $f b$} & \multirow{2}{*}{ Points } \\
\cline { 4 - 5 } & in $f b$ & after & after & Total SS3OS11 \\
& & & $D 1$ & $D 2$ & events \\
\hline \multirow{5}{*}{ LHLS } & BP1 & 210.1 & 0.05 & 0.04 & 3.99 \\
& BP2 & 131.5 & 0.03 & 0.028 & 2.36 \\
\cline { 3 - 5 } & BP1-DM & 151.8 & 0.04 & 0.03 & 2.88 \\
& BP2-DM & 45.25 & 0.013 & 0.012 & 1.18 \\
\hline
\end{tabular}

Table 16. The production cross sections of all EWeakino pairs and $\sigma_{\text {eff }}^{s s 3 o s 1}$ after successive cuts for the BPs defined in table 6 and table 8. Also the total number of SS3OS1l events are shown for an integrated luminosity of $100 \mathrm{fb}^{-1}$. 


\begin{tabular}{|c|c|c|c|}
\hline Background & \multirow{2}{*}{$\sigma_{\text {prod }}$} & \multicolumn{2}{|c|}{$\sigma_{\text {eff }}^{5 l}$ in $f b$} \\
\cline { 3 - 4 } Processes & in $p b$ & after & after \\
& & $D 1$ & $D 2$ \\
\hline$t \bar{t} Z$ & 0.018 & 0.002 & 0.0007 \\
$W Z Z$ & 0.042 & 0.013 & 0.005 \\
$Z Z Z$ & 0.010 & 0.001 & 0.0003 \\
\hline
\end{tabular}

Table 17. The production level and effective cross-sections $\left(\sigma_{\text {eff }}^{5 l}\right)$ after the cuts of different backgrounds.

\begin{tabular}{|c|c|c|c|c|c|}
\hline \multirow[b]{2}{*}{ Models } & \multirow{2}{*}{$\begin{array}{c}\text { Benchmark } \\
\text { Points }\end{array}$} & \multirow{2}{*}{$\begin{array}{l}\sigma_{\text {prod }} \\
\text { in } f b\end{array}$} & \multicolumn{2}{|c|}{$\sigma_{\mathrm{eff}}^{5 l}$ in $f b$} & \multirow[b]{2}{*}{$\begin{array}{c}\text { Total } 5 l \\
\text { events }\end{array}$} \\
\hline & & & $\begin{array}{c}\text { after } \\
D 1\end{array}$ & $\begin{array}{c}\text { after } \\
D 2\end{array}$ & \\
\hline \multirow{6}{*}{ Compressed } & BP1 & 507.9 & 0.16 & 0.096 & 9.65 \\
\hline & BP2 & 650.2 & 0.06 & 0.052 & 5.20 \\
\hline & BP3 & 1417. & 0.03 & 0.028 & 2.83 \\
\hline & $\mathrm{BP} 4$ & 1763. & 0.02 & 0.017 & 1.76 \\
\hline & BP1-DM & 56.38 & 0.014 & 0.012 & 1.20 \\
\hline & BP2-DM & 2817. & 0.03 & 0.028 & 2.81 \\
\hline \multirow{8}{*}{ LHHS } & BP1 & 555.4 & 0.11 & 0.077 & 7.77 \\
\hline & $\mathrm{BP} 2$ & 301.4 & 0.05 & 0.039 & 3.91 \\
\hline & BP3 & 200.5 & 0.02 & 0.018 & 1.80 \\
\hline & $\mathrm{BP} 4$ & 92.56 & 0.013 & 0.012 & 1.20 \\
\hline & BP1-DM & 370.8 & 0.06 & 0.041 & 4.10 \\
\hline & BP2-DM & 156.3 & 0.025 & 0.015 & 1.56 \\
\hline & BP3-DM & 134.8 & 0.011 & 0.011 & 1.13 \\
\hline & BP4-DM & 71.12 & 0.016 & 0.014 & 1.42 \\
\hline
\end{tabular}

Table 18. The production cross sections of all EWeakino pairs and $\sigma_{\text {eff }}^{5 l}$ for the BPs defined in table 4 and table 6. Also the total number of $5 l$ events are shown for an integrated luminosity of $100 \mathrm{fb}^{-1}$.

bring it down to a negligible level. The effect of the cuts on the SM processes is illustrated in table 17 .

We quote the number of signal events in table 18-19.

Note that in this case signal events in WMAP/PLANCK allowed points never reach 5 for $100 \mathrm{fb}^{-1}$ of integrated luminosity though some of them may show up as a hint in the early phases of RUN II. Therefore, one has to wait for upgradation in luminosity to claim a discovery through this channel. 


\begin{tabular}{|c|c|c|c|c|c|}
\hline \multirow[b]{2}{*}{ Models } & \multirow{2}{*}{$\begin{array}{c}\text { Benchmark } \\
\text { Points }\end{array}$} & \multirow{2}{*}{$\begin{array}{l}\sigma_{\text {prod }} \\
\text { in } f b\end{array}$} & \multicolumn{2}{|c|}{$\sigma_{\text {eff }}^{5 l}$ in $f b$} & \multirow[b]{2}{*}{$\begin{array}{l}\text { Total } 5 l \\
\text { events }\end{array}$} \\
\hline & & & $\begin{array}{c}\text { after } \\
D 1\end{array}$ & $\begin{array}{c}\text { after } \\
D 2\end{array}$ & \\
\hline \multirow{5}{*}{ LHLS } & BP1 & 210.1 & 0.039 & 0.035 & 3.57 \\
\hline & BP2 & 131.5 & 0.025 & 0.018 & 1.84 \\
\hline & BP3 & 69.93 & 0.012 & 0.01 & 1.04 \\
\hline & BP1-DM & 151.8 & 0.044 & 0.036 & 3.64 \\
\hline & BP2-DM & 45.25 & 0.014 & 0.012 & 1.08 \\
\hline
\end{tabular}

Table 19. The production cross-sections of all EWeakino pairs and $\sigma_{\text {eff }}^{5 l}$ for the BPs defined in table 5 and table 7 . Also the total number of $5 l$ events are shown for an integrated luminosity of $100 f b^{-1}$.

\begin{tabular}{|c|c|c|c|}
\hline Points & $\mu=x M_{1}$ & $\tilde{\chi}_{1}^{0}=200, \tilde{\chi}_{2}^{ \pm}=400$ & $\Omega_{\tilde{\chi}}$ \\
\hline 1 & $x=1.05$ & $\tilde{\chi}_{1}^{ \pm}=231$ & 0.0238 \\
2 & $x=1.20$ & $\tilde{\chi}_{1}^{ \pm}=248$ & 0.0566 \\
3 & $x=1.30$ & $\tilde{\chi}_{1}^{ \pm}=260$ & 0.0975 \\
\hline Points & $\mu=x M_{1}$ & $\widetilde{\chi}_{1}^{0}=300, \widetilde{\chi}_{2}^{ \pm}=500$ & $\Omega_{\tilde{\chi}}$ \\
\hline 4 & $x=1.05$ & $\tilde{\chi}_{1}^{ \pm}=335$ & 0.044 \\
5 & $x=1.10$ & $\tilde{\chi}_{1}^{ \pm}=342$ & 0.0669 \\
6 & $x=1.15$ & $\tilde{\chi}_{1}^{ \pm}=350$ & 0.102 \\
\hline
\end{tabular}

Table 20. DM relic densities for two different $\widetilde{\chi}_{2}^{ \pm}$masses in relaxed compression scenario. All masses are in $\mathrm{GeV}$.

\subsection{Multilepton signals in moderately compressed LHHS models}

As already discussed in section 2.1 and confirmed in section 6.1, there is a tension between the DM relic density constraint and low $m_{\tilde{\chi}_{2}^{ \pm}}$in a highly compressed scenario characterised by the representative choice $\mu=1.05 M_{1}$. Relaxing the degree of compression one obtains consistency with the observed DM relic density for much lower $m_{\widetilde{\chi}_{2}^{ \pm}}$(see table 20).

It follows from table 9 that the parameter space consistent with the DM relic density data may not yield a $3 l+\mathbb{E}_{T}$ signal. On the other hand for $\mu=1.3 M_{1}$ it follows from table 21 that encouraging $3 l$ signals are predicted in all cases. Other background free multilepton signals also look promising.

\subsection{Discriminating different pMSSM models via multilepton signatures}

As pointed out in [52] if more than one multilepton signatures show up before the next long shutdown of the LHC, their relative rates may distinguish different models studied in this paper ${ }^{10}$ This can be showcased by the compressed model. For BP1-DM, BP2-DM and

\footnotetext{
${ }^{10}$ In [53] the possibility of distinguishing between the MSSM and one scenario in the TMSSM i.e. the MSSM supplemented by one hyperchargeless $\mathrm{SU}(2)_{L}$-triplet chiral superfield $[113,114]$ was explored.
} 


\begin{tabular}{|c|c|c|c|c|c|c|c|}
\hline & \multicolumn{3}{|c|}{ Masses } & \multicolumn{4}{c|}{ Signals } \\
\cline { 2 - 8 } Points & $\widetilde{\chi}_{1}^{0}$ & $\widetilde{\chi}_{1}^{ \pm}$ & $\widetilde{\chi}_{2}^{ \pm}$ & $(S / \sqrt{B})_{3 l}$ & 41 & SS3OS1l & 51 \\
\hline 1 & 200 & 265 & 420 & 6.2 & 22.5 & 9.6 & 5.3 \\
2 & 200 & 275 & 500 & 7.2 & 13.7 & 3.5 & 4.1 \\
3 & 250 & 300 & 420 & 3.7 & 15.7 & 4.6 & 6.1 \\
\hline
\end{tabular}

Table 21. Number of events surviving all cuts for all types of signals for an integrated luminosity of $100 \mathrm{fb}^{-1}$ in the moderately compressed model. Masses are given in GeV.

BP3-DM the $3 l$ signal is unobservable whereas for the first and the last BP the $4 l$ signal is likely to be observed. It is interesting to note that for all other BPs the 31 signal is observable. Thus if the $4 \mathrm{l}$ signal (and not the 31 signal) is observed BP1-DM and BP3-DM would be strong candidates for the underlying physics. These two models, in turn, can be distinguished since only for BP3-DM the SS3OS1l signal is observable.

In fact the SS3OS1l signal could be a useful discriminator for the models. Both the 31 and SS3OS1l signals can be observed in the compressed model (BP1, BP2) and the LHHS model (BP1). The ratio $r_{s s / 4 l}=$ (the number of SS3OS1l events) / (the number of $4 \mathrm{l}$ events) is approximately $1 / 3(1 / 2)$ for LHHS (BP1) (compressed(BP2)) model. On the other hand for compressed model (BP1) the ratio $r_{4 l / 3 l}=$ (the number of 41 events) $/$ (the number of 31 events) is roughly 1.4 while the same ratio is significantly smaller than 1 for compressed (BP2) and LHHS (BP1).

This procedure can be employed for distinguishing the BPs presented in tables 4-7 from each other. Obviously the method will be more efficient as luminosity accumulates at the LHC and reduces the statistical errors. Some of the systematics like uncertainties in the production cross-sections cancel if we consider the relative rates.

\section{Conclusion}

In order to extend and complement [52], we have examined the complete EWeakino sector of several pMSSM models with our main attention focused on the heavier EWeakinos.

In section 2 we have argued that the $3 l+\mathbb{E}_{T}$ data allows models with relatively light Eweakinos provided the heavier EWeakinos are wino like, the lighter ones are higgsino like (i.e., $M_{2}>\mu$ ). On the other hand naturalness arguments generically favour small $\mu$. LSP can be bino like or a bino-higgsino admixture. The latter choice has been advocated by many since it is compatible with both the observed DM relic density and the naturalness condition. Accordingly we have targeted the compressed model (section 2.1), the LHHS model (section 2.2), the LHLS model (section 2.3) and the LMLS model (section 2.4). In all cases either the heavier EWeakinos or both lighter and heavier EWeakinos directly decay into on shell sleptons.

In section 3 we have computed the production cross-sections of all EWeakinos pairs and the BRs of each EWeakino in the above models to illustrate that the multilepton $(m l)$ 
$+\mathbb{E}_{T}$ final states for $m>3$ are viable only if the heavier EWeakinos are not decoupled (See table 3).

In section 4 we describe our methodology. The constraints that we used in our analyses are summarized in section 4.1. We do not consider some often used constraints, most notably the direct DM detection data, since they involve sizable uncertainties. However, we have shown in the subsequent sections that the models studied by us are compatible with the data if allowances are made for these uncertainties.

In section 5 we delineated the allowed parameter space in each case subject to the constraints from the LHC $3 l+\mathbb{E}_{T}$ data, the observed DM relic density of the universe and the precisely measured anomalous magnetic moment of the muon at the $\lesssim 2 \sigma$ level (See figures 1-4). The largest parameter space is allowed in the compressed model $(\mu=$ $\left.1.05 M_{1}\right)$. However, consistency with the DM relic density constraint requires that $m_{\widetilde{\chi}_{2}^{ \pm}} \geq$ $600 \mathrm{GeV}$ irrespective of $m_{\widetilde{\chi}_{1}^{0}}$. This may adversely affect the observability of some potential multilepton signatures. If the compression is relaxed lower values of $m_{\widetilde{\chi}_{2}^{ \pm}}$are allowed.

In section 6 we select benchmark points (BPs) from the APS of each model delineated in section 5 (See figures 1-4). We show that in most cases observable $3 l$ (section 6.1), $4 l$ (section 6.2), SS3OS1l (section 6.3) and $5 l$ (section 6.4) signal accompanied by large $\mathbb{E}_{T}$ can all show up before the next long shut down of the LHC. None of the signals are viable if the heavier EWeakinos are decoupled.

We show in section 6.5 that if the compression is relaxed, smaller $m_{\widetilde{\chi}_{2}^{ \pm}}(\lesssim 600 \mathrm{GeV})$ is compatible with DM relic density constraint (See table 20) and observable multilepton signals are viable (table 21 ).

In section 6.6 we discuss the prospect of discriminating between competing models using the relative rates of different multilepton signatures in these models.

\section{Acknowledgments}

MC would like to thank TRR33 "The Dark Universe" project for financial support. NG thanks Science and Engineering Research Board, Department of Science and Technology, India for a research fellowship.

Open Access. This article is distributed under the terms of the Creative Commons Attribution License (CC-BY 4.0), which permits any use, distribution and reproduction in any medium, provided the original author(s) and source are credited.

\section{References}

[1] H.P. Nilles, Supersymmetry, supergravity and particle physics, Phys. Rept. 110 (1984) 1 [INSPIRE].

[2] H.E. Haber and G.L. Kane, The search for supersymmetry: probing physics beyond the Standard Model, Phys. Rept. 117 (1985) 75 [INSPIRE].

[3] D.J.H. Chung, L.L. Everett, G.L. Kane, S.F. King, J.D. Lykken and L.-T. Wang, The soft supersymmetry breaking Lagrangian: theory and applications, Phys. Rept. 407 (2005) 1 [hep-ph/0312378] [INSPIRE]. 
[4] S.P. Martin, A supersymmetry primer, Adv. Ser. Direct. High Energy Phys. 18 (1998) 1 [Adv. Ser. Direct. High Energy Phys. 21 (2010) 1] [hep-ph/9709356] [InSPIRE].

[5] M. Drees, P. Roy and R.M. Godbole, Theory and phenomenology of sparticles, World Scientific, Singapore, (2005).

[6] H. Baer and X. Tata, Weak scale supersymmetry: from superfields to scattering events, Cambridge Univ. Pr., Cambridge U.K., (2006).

[7] ATLAS supersymmetry searches public results webpage, https://twiki.cern.ch/twiki/bin/view/AtlasPublic/SupersymmetryPublicResults.

[8] CMS supersymmetry physics results webpage, https://twiki.cern.ch/twiki/bin/view/CMSPublic/PhysicsResultsSUS.

[9] WMAP collaboration, G. Hinshaw et al., Nine-year Wilkinson Microwave Anisotropy Probe (WMAP) observations: cosmological parameter results, Astrophys. J. Suppl. 208 (2013) 19 [arXiv:1212 .5226] [INSPIRE].

[10] Planck collaboration, P.A.R. Ade et al., Planck 2015 results. XIII. Cosmological parameters, Astron. Astrophys. 594 (2016) A13 [arXiv: 1502.01589] [INSPIRE].

[11] K.A. Olive, TASI lectures on astroparticle physics, astro-ph/0503065 [INSPIRE].

[12] H. Baer and X. Tata, Dark matter and the LHC, in Physics at the Large Hadron Collider, Indian National Science Academy, A Platinum Jubilee Special Issue, A. Datta, B. Mukhopadhyaya and A. Raychaudhuri eds., Springer, New Delhi India, (2009) [arXiv:0805.1905].

[13] S. Arrenberg et al., Working group report: dark matter complementarity, arXiv:1310.8621 [INSPIRE].

[14] G. Bélanger, F. Boudjema, A. Cottrant, R.M. Godbole and A. Semenov, The MSSM invisible Higgs in the light of dark matter and $g$-2, Phys. Lett. B 519 (2001) 93 [hep-ph/0106275] [INSPIRE].

[15] U. Chattopadhyay, D. Das, A. Datta and S. Poddar, Non-zero trilinear parameter in the mSUGRA model: dark matter and collider signals at Tevatron and LHC, Phys. Rev. D 76 (2007) 055008 [arXiv:0705.0921] [INSPIRE].

[16] M. Abdughani, L. Wu and J.M. Yang, The mixed bino-higgsino dark matter in natural SUSY confronted with XENON1T/PandaX and LHC data, arXiv:1705.09164 [INSPIRE].

[17] L. Roszkowski, E.M. Sessolo and A.J. Williams, Prospects for dark matter searches in the pMSSM, JHEP 02 (2015) 014 [arXiv:1411.5214] [INSPIRE].

[18] J. Bramante et al., Relic neutralino surface at a $100 \mathrm{TeV}$ collider, Phys. Rev. D 91 (2015) 054015 [arXiv: 1412.4789] [INSPIRE].

[19] A. Choudhury, K. Kowalska, L. Roszkowski, E.M. Sessolo and A.J. Williams, Less-simplified models of dark matter for direct detection and the LHC, JHEP 04 (2016) 182 [arXiv: 1509.05771] [INSPIRE].

[20] J. Bramante, N. Desai, P. Fox, A. Martin, B. Ostdiek and T. Plehn, Towards the final word on neutralino dark matter, Phys. Rev. D 93 (2016) 063525 [arXiv:1510.03460] [INSPIRE].

[21] K. Hamaguchi and K. Ishikawa, Prospects for Higgs- and Z-resonant neutralino dark matter, Phys. Rev. D 93 (2016) 055009 [arXiv: 1510.05378] [inSPIRE]. 
[22] J. Cao, Y. He, L. Shang, W. Su and Y. Zhang, Testing the light dark matter scenario of the MSSM at the LHC, JHEP 03 (2016) 207 [arXiv: 1511.05386] [INSPIRE].

[23] U. Chattopadhyay and A. Dey, Probing non-holomorphic MSSM via precision constraints, dark matter and LHC data, JHEP 10 (2016) 027 [arXiv:1604.06367] [INSPIRE].

[24] M. Chakraborti, U. Chattopadhyay and S. Poddar, How light a higgsino or a wino dark matter can become in a compressed scenario of MSSM, JHEP 09 (2017) 064 [arXiv: 1702.03954] [INSPIRE].

[25] A. Arbey, M. Boudaud, F. Mahmoudi and G. Robbins, Robustness of dark matter constraints and interplay with collider searches for new physics, arXiv:1707.00426 [INSPIRE].

[26] C. Boehm, A. Djouadi and M. Drees, Light scalar top quarks and supersymmetric dark matter, Phys. Rev. D 62 (2000) 035012 [hep-ph/9911496] [INSPIRE].

[27] J.R. Ellis, K.A. Olive and Y. Santoso, Calculations of neutralino stop coannihilation in the CMSSM, Astropart. Phys. 18 (2003) 395 [hep-ph/0112113] [INSPIRE].

[28] A. Chatterjee, A. Choudhury, A. Datta and B. Mukhopadhyaya, Gluino mass limits with sbottom NLSP in coannihilation scenarios, JHEP 01 (2015) 154 [arXiv:1411.6467] [INSPIRE].

[29] G. Bélanger, D. Ghosh, R. Godbole and S. Kulkarni, Light stop in the MSSM after LHC run 1, JHEP 09 (2015) 214 [arXiv: 1506.00665] [INSPIRE].

[30] B. Kaufman, P. Nath, B.D. Nelson and A.B. Spisak, Light stops and observation of supersymmetry at LHC run 2, Phys. Rev. D 92 (2015) 095021 [arXiv:1509. 02530] [INSPIRE].

[31] P. Nath and A.B. Spisak, Gluino coannihilation and observability of gluinos at LHC run 2, Phys. Rev. D 93 (2016) 095023 [arXiv: 1603.04854] [InSPIRE].

[32] Muon G-2 collaboration, G.W. Bennett et al., Final report of the muon E821 anomalous magnetic moment measurement at BNL, Phys. Rev. D 73 (2006) 072003 [hep-ex/0602035] [INSPIRE].

[33] B.L. Roberts, Status of the Fermilab muon $(g-2)$ experiment, Chin. Phys. C 34 (2010) 741 [arXiv: 1001.2898] [INSPIRE].

[34] F. Jegerlehner and A. Nyffeler, The muon g-2, Phys. Rept. 477 (2009) 1 [arXiv: 0902.3360] [INSPIRE].

[35] K. Hagiwara, R. Liao, A.D. Martin, D. Nomura and T. Teubner, $(g-2)_{\mu}$ and $\alpha\left(M_{Z}^{2}\right)$ re-evaluated using new precise data, J. Phys. G 38 (2011) 085003 [arXiv:1105.3149] [INSPIRE].

[36] N. Sakai, Naturalness in supersymmetric GUTs, Z. Phys. C 11 (1981) 153 [INSPIRE].

[37] R.K. Kaul and P. Majumdar, Cancellation of quadratically divergent mass corrections in globally supersymmetric spontaneously broken gauge theories, Nucl. Phys. B 199 (1982) 36 [INSPIRE].

[38] R. Barbieri and G.F. Giudice, Upper bounds on supersymmetric particle masses, Nucl. Phys. B 306 (1988) 63 [INSPIRE].

[39] J.L. Feng, Naturalness and the status of supersymmetry, Ann. Rev. Nucl. Part. Sci. 63 (2013) 351 [arXiv: 1302.6587] [INSPIRE]. 
[40] H. Baer, A. Mustafayev, E.-K. Park and X. Tata, Target dark matter detection rates in models with a well-tempered neutralino, JCAP 01 (2007) 017 [hep-ph/0611387] [INSPIRE].

[41] J.A. Casas, J.M. Moreno, S. Robles, K. Rolbiecki and B. Zaldívar, What is a natural SUSY scenario?, JHEP 06 (2015) 070 [arXiv: 1407.6966] [INSPIRE].

[42] ATLAS collaboration, Search for direct production of charginos and neutralinos in events with three leptons and missing transverse momentum in $\sqrt{s}=8 \mathrm{TeV}$ pp collisions with the ATLAS detector, JHEP 04 (2014) 169 [arXiv: 1402.7029] [INSPIRE].

[43] CMS collaboration, Searches for electroweak neutralino and chargino production in channels with Higgs, $Z$ and $W$ bosons in pp collisions at 8 TeV, Phys. Rev. D 90 (2014) 092007 [arXiv: 1409.3168] [INSPIRE].

[44] M. Chakraborti, U. Chattopadhyay, A. Choudhury, A. Datta and S. Poddar, The electroweak sector of the pMSSM in the light of LHC $-8 \mathrm{TeV}$ and other data, JHEP 07 (2014) 019 [arXiv: 1404.4841] [inSPIRE].

[45] M. Chakraborti, U. Chattopadhyay, A. Choudhury, A. Datta and S. Poddar, Reduced LHC constraints for higgsino-like heavier electroweakinos, JHEP 11 (2015) 050 [arXiv: 1507.01395] [INSPIRE].

[46] A. Choudhury and A. Datta, Neutralino dark matter confronted by the LHC constraints on electroweak SUSY signals, JHEP 09 (2013) 119 [arXiv: 1305.0928] [INSPIRE].

[47] J. Eckel, M.J. Ramsey-Musolf, W. Shepherd and S. Su, Impact of LSP character on slepton reach at the LHC, JHEP 11 (2014) 117 [arXiv:1408.2841] [INSPIRE].

[48] C. Han, Probing light bino and higgsinos at the LHC, arXiv:1409.7000 [INSPIRE].

[49] C. Han, L. Wu, J.M. Yang, M. Zhang and Y. Zhang, New approach for detecting a compressed bino/wino at the LHC, Phys. Rev. D 91 (2015) 055030 [arXiv:1409.4533] [INSPIRE].

[50] R.K. Barman, B. Bhattacherjee, A. Chakraborty and A. Choudhury, Study of MSSM heavy Higgs bosons decaying into charginos and neutralinos, Phys. Rev. D 94 (2016) 075013 [arXiv: 1607.00676] [INSPIRE].

[51] T.A.W. Martin and D. Morrissey, Electroweakino constraints from LHC data, JHEP 12 (2014) 168 [arXiv: 1409.6322] [INSPIRE].

[52] A. Datta, N. Ganguly and S. Poddar, New limits on heavier electroweakinos and their LHC signatures, Phys. Lett. B 763 (2016) 213 [arXiv:1606.04391] [INSPIRE].

[53] C. Arina, M. Chala, V. Martin-Lozano and G. Nardini, Confronting SUSY models with LHC data via electroweakino production, JHEP 12 (2016) 149 [arXiv:1610.03822] [INSPIRE].

[54] Planck collaboration, P.A.R. Ade et al., Planck 2013 results. XVI. Cosmological parameters, Astron. Astrophys. 571 (2014) A16 [arXiv:1303.5076] [INSPIRE].

[55] MSSM Working Group collaboration, A. Djouadi et al., The minimal supersymmetric Standard Model: group summary report, hep-ph/9901246 [INSPIRE].

[56] A. Djouadi, The anatomy of electro-weak symmetry breaking II. The Higgs bosons in the minimal supersymmetric model, Phys. Rept. 459 (2008) 1 [hep-ph/0503173] [INSPIRE].

[57] P. Bechtle et al., The light and heavy Higgs interpretation of the MSSM, Eur. Phys. J. C 77 (2017) 67 [arXiv: 1608.00638] [INSPIRE]. 
[58] N. Arkani-Hamed, A. Delgado and G.F. Giudice, The well-tempered neutralino, Nucl. Phys. B 741 (2006) 108 [hep-ph/0601041] [INSPIRE].

[59] T. Sjöstrand, S. Mrenna and P.Z. Skands, PYTHIA 6.4 physics and manual, JHEP 05 (2006) 026 [hep-ph/0603175] [INSPIRE].

[60] ATLAS collaboration, Search for direct production of charginos, neutralinos and sleptons in final states with two leptons and missing transverse momentum in pp collisions at $\sqrt{s}=8 \mathrm{TeV}$ with the ATLAS detector, JHEP 05 (2014) 071 [arXiv:1403.5294] [INSPIRE].

[61] S.P. Das, M. Guchait and D.P. Roy, Testing SUSY models for the muon g- 2 anomaly via chargino-neutralino pair production at the LHC, Phys. Rev. D 90 (2014) 055011 [arXiv:1406.6925] [INSPIRE].

[62] K. Kowalska, L. Roszkowski, E.M. Sessolo and A.J. Williams, GUT-inspired SUSY and the muon g- 2 anomaly: prospects for LHC 14 TeV, JHEP 06 (2015) 020 [arXiv:1503.08219] [INSPIRE].

[63] J. Chakrabortty, A. Choudhury and S. Mondal, Non-universal gaugino mass models under the lamppost of muon $(g-2)$, JHEP 07 (2015) 038 [arXiv: 1503.08703] [INSPIRE].

[64] A. Choudhury, L. Darmé, L. Roszkowski, E.M. Sessolo and S. Trojanowski, Muon g-2 and related phenomenology in constrained vector-like extensions of the MSSM, JHEP 05 (2017) 072 [arXiv: 1701.08778] [INSPIRE].

[65] M. Endo, K. Hamaguchi, S. Iwamoto and K. Yanagi, Probing minimal SUSY scenarios in the light of muon $g-2$ and dark matter, JHEP 06 (2017) 031 [arXiv:1704.05287] [INSPIRE].

[66] K. Hagiwara, K. Ma and S. Mukhopadhyay, Closing in on the chargino contribution to the muon $g-2$ in the MSSM: current LHC constraints, arXiv:1706.09313 [INSPIRE].

[67] ATLAS collaboration, Observation of a new particle in the search for the Standard Model Higgs boson with the ATLAS detector at the LHC, Phys. Lett. B 716 (2012) 1 [arXiv:1207.7214] [INSPIRE].

[68] CMS collaboration, Observation of a new boson at a mass of $125 \mathrm{GeV}$ with the CMS experiment at the LHC, Phys. Lett. B 716 (2012) 30 [arXiv:1207.7235] [INSPIRE].

[69] G. Degrassi, S. Heinemeyer, W. Hollik, P. Slavich and G. Weiglein, Towards high precision predictions for the MSSM Higgs sector, Eur. Phys. J. C 28 (2003) 133 [hep-ph/0212020] [INSPIRE].

[70] B.C. Allanach, A. Djouadi, J.L. Kneur, W. Porod and P. Slavich, Precise determination of the neutral Higgs boson masses in the MSSM, JHEP 09 (2004) 044 [hep-ph/0406166] [INSPIRE].

[71] S.P. Martin, Three-loop corrections to the lightest Higgs scalar boson mass in supersymmetry, Phys. Rev. D 75 (2007) 055005 [hep-ph/0701051] [INSPIRE].

[72] R.V. Harlander, P. Kant, L. Mihaila and M. Steinhauser, Higgs boson mass in supersymmetry to three loops, Phys. Rev. Lett. 100 (2008) 191602 [Erratum ibid. 101 (2008) 039901] [arXiv:0803.0672] [INSPIRE].

[73] S. Heinemeyer, O. Stal and G. Weiglein, Interpreting the LHC Higgs search results in the MSSM, Phys. Lett. B 710 (2012) 201 [arXiv:1112.3026] [INSPIRE]. 
[74] A. Arbey, M. Battaglia, A. Djouadi and F. Mahmoudi, The Higgs sector of the phenomenological MSSM in the light of the Higgs boson discovery, JHEP 09 (2012) 107 [arXiv:1207.1348] [INSPIRE].

[75] J.L. Lopez, D.V. Nanopoulos and X. Wang, Large $(g-2)_{\mu}$ in $\mathrm{SU}(5) \times \mathrm{U}(1)$ supergravity models, Phys. Rev. D 49 (1994) 366 [hep-ph/9308336] [InSPIRE].

[76] U. Chattopadhyay and P. Nath, Probing supergravity grand unification in the Brookhaven g- 2 experiment, Phys. Rev. D 53 (1996) 1648 [hep-ph/9507386] [INSPIRE].

[77] T. Moroi, The muon anomalous magnetic dipole moment in the minimal supersymmetric Standard Model, Phys. Rev. D 53 (1996) 6565 [Erratum ibid. D 56 (1997) 4424] [hep-ph/9512396] [INSPIRE].

[78] S. Heinemeyer, D. Stöckinger and G. Weiglein, Two loop SUSY corrections to the anomalous magnetic moment of the muon, Nucl. Phys. B 690 (2004) 62 [hep-ph/0312264] [InSPIRE].

[79] G.-C. Cho, K. Hagiwara, Y. Matsumoto and D. Nomura, The MSSM confronts the precision electroweak data and the muon $g-2$, JHEP 11 (2011) 068 [arXiv:1104.1769] [INSPIRE].

[80] M. Endo, K. Hamaguchi, S. Iwamoto and T. Yoshinaga, Muon g- 2 vs LHC in supersymmetric models, JHEP 01 (2014) 123 [arXiv:1303.4256] [INSPIRE].

[81] S. Akula, P. Nath and G. Peim, Implications of the Higgs boson discovery for mSUGRA, Phys. Lett. B 717 (2012) 188 [arXiv:1207.1839] [INSPIRE].

[82] H. Baer, V. Barger, P. Huang, D. Mickelson, A. Mustafayev and X. Tata, Post-LHC7 fine-tuning in the minimal supergravity/CMSSM model with a $125 \mathrm{GeV}$ Higgs boson, Phys. Rev. D 87 (2013) 035017 [arXiv:1210.3019] [INSPIRE].

[83] S. Mohanty, S. Rao and D.P. Roy, Reconciling the muon $g-2$ and dark matter relic density with the LHC results in nonuniversal gaugino mass models, JHEP 09 (2013) 027 [arXiv: 1303.5830] [INSPIRE].

[84] S. Akula and P. Nath, Gluino-driven radiative breaking, Higgs boson mass, muon $g-2$ and the Higgs diphoton decay in supergravity unification, Phys. Rev. D 87 (2013) 115022 [arXiv: 1304.5526] [INSPIRE].

[85] N. Okada, S. Raza and Q. Shafi, Particle spectroscopy of supersymmetric SU(5) in light of 125 GeV Higgs and muon $g$ - 2 data, Phys. Rev. D 90 (2014) 015020 [arXiv:1307.0461] [INSPIRE].

[86] J. Chakrabortty, S. Mohanty and S. Rao, Non-universal gaugino mass GUT models in the light of dark matter and LHC constraints, JHEP 02 (2014) 074 [arXiv:1310.3620] [INSPIRE].

[87] J. Kozaczuk and S. Profumo, Light NMSSM neutralino dark matter in the wake of CDMS II and a $126 \mathrm{GeV}$ Higgs boson, Phys. Rev. D 89 (2014) 095012 [arXiv:1308.5705] [InSPIRE].

[88] G. Bertone et al., Global analysis of the pMSSM in light of the Fermi GeV excess: prospects for the LHC run 2 and astroparticle experiments, JCAP 04 (2016) 037 [arXiv: 1507.07008] [INSPIRE].

[89] M. Badziak, M. Olechowski and P. Szczerbiak, Spin-dependent constraints on blind spots for thermal singlino-higgsino dark matter with(out) light singlets, JHEP 07 (2017) 050 [arXiv: 1705.00227] [INSPIRE]. 
[90] J. Harz, B. Herrmann, M. Klasen, K. Kovarik and P. Steppeler, Theoretical uncertainty of the supersymmetric dark matter relic density from scheme and scale variations, Phys. Rev. D 93 (2016) 114023 [arXiv: 1602.08103] [INSPIRE].

[91] LUX collaboration, D.S. Akerib et al., Results from a search for dark matter in the complete LUX exposure, Phys. Rev. Lett. 118 (2017) 021303 [arXiv:1608. 07648] [InSPIRE].

[92] J.I. Read, The local dark matter density, J. Phys. G 41 (2014) 063101 [arXiv:1404.1938] [INSPIRE].

[93] C. Cheung, L.J. Hall, D. Pinner and J.T. Ruderman, Prospects and blind spots for neutralino dark matter, JHEP 05 (2013) 100 [arXiv:1211.4873] [INSPIRE].

[94] LUX collaboration, D.S. Akerib et al., First results from the LUX dark matter experiment at the Sanford Underground Research Facility, Phys. Rev. Lett. 112 (2014) 091303 [arXiv:1310.8214] [INSPIRE].

[95] M. Klasen, M. Pohl and G. Sigl, Indirect and direct search for dark matter, Prog. Part. Nucl. Phys. 85 (2015) 1 [arXiv:1507.03800] [inSPIRE].

[96] L. Baudis, Dark matter searches, Annalen Phys. 528 (2016) 74 [arXiv:1509.00869] [INSPIRE].

[97] ATLAS collaboration, Search for direct production of charginos and neutralinos in events with three leptons and missing transverse momentum in $21 \mathrm{fb}^{-1}$ of pp collisions at $\sqrt{s}=8 \mathrm{TeV}$ with the ATLAS detector, ATLAS-CONF-2013-035, CERN, Geneva Switzerland, (2013).

[98] A. Choudhury and S. Mondal, Revisiting the exclusion limits from direct chargino-neutralino production at the LHC, Phys. Rev. D 94 (2016) 055024 [arXiv: 1603.05502] [INSPIRE].

[99] W. Beenakker, R. Hopker and M. Spira, PROSPINO: a program for the production of supersymmetric particles in next-to-leading order QCD, hep-ph/9611232 [INSPIRE].

[100] M.L. Mangano, M. Moretti, F. Piccinini, R. Pittau and A.D. Polosa, ALPGEN, a generator for hard multiparton processes in hadronic collisions, JHEP 07 (2003) 001 [hep-ph/0206293] [INSPIRE].

[101] M.L. Mangano, M. Moretti, F. Piccinini and M. Treccani, Matching matrix elements and shower evolution for top-quark production in hadronic collisions, JHEP 01 (2007) 013 [hep-ph/0611129] [INSPIRE].

[102] S. Hoeche et al., Matching parton showers and matrix elements, hep-ph/0602031 [INSPIRE].

[103] M. Cacciari, G.P. Salam and G. Soyez, The anti-k $k_{t}$ jet clustering algorithm, JHEP 04 (2008) 063 [arXiv:0802.1189] [INSPIRE].

[104] M. Cacciari, G.P. Salam and G. Soyez, FastJet user manual, Eur. Phys. J. C 72 (2012) 1896 [arXiv: 1111.6097] [INSPIRE].

[105] J. Pumplin, D.R. Stump, J. Huston, H.L. Lai, P.M. Nadolsky and W.K. Tung, New generation of parton distributions with uncertainties from global QCD analysis, JHEP $\mathbf{0 7}$ (2002) 012 [hep-ph/0201195] [INSPIRE].

[106] A. Djouadi, J.-L. Kneur and G. Moultaka, SuSpect: a Fortran code for the supersymmetric and Higgs particle spectrum in the MSSM, Comput. Phys. Commun. 176 (2007) 426 [hep-ph/0211331] [INSPIRE]. 
[107] A. Djouadi, M.M. Muhlleitner and M. Spira, Decays of supersymmetric particles: the program SUSY-HIT (SUspect-SdecaY-HDECAY-InTerface), Acta Phys. Polon. B 38 (2007) 635 [hep-ph/0609292] [INSPIRE].

[108] G. Bélanger, F. Boudjema, A. Pukhov and A. Semenov, MicrOMEGAss : a program for calculating dark matter observables, Comput. Phys. Commun. 185 (2014) 960 [arXiv: 1305.0237] [INSPIRE].

[109] LEP SUSY working group webpage, http://lepsusy.web.cern.ch/lepsusy/.

[110] M. Badziak, M. Olechowski and P. Szczerbiak, Is well-tempered neutralino in MSSM still alive after 2016 LUX results?, Phys. Lett. B 770 (2017) 226 [arXiv:1701. 05869] [INSPIRE].

[111] ATLAS collaboration, Measurement of the b-tag efficiency in a sample of jets containing muons with $5 \mathrm{fb}^{-1}$ of data from the ATLAS detector, ATLAS-CONF-2012-043, CERN, Geneva Switzerland, (2012).

[112] ATLAS collaboration, Search for supersymmetry in events with four or more leptons in $\sqrt{s}=8 \mathrm{TeV}$ pp collisions with the ATLAS detector, Phys. Rev. D 90 (2014) 052001 [arXiv: 1405.5086] [INSPIRE].

[113] J.R. Espinosa and M. Quirós, Higgs triplets in the supersymmetric Standard Model, Nucl. Phys. B 384 (1992) 113 [INSPIRE].

[114] S. Di Chiara and K. Hsieh, Triplet extended supersymmetric Standard Model, Phys. Rev. D 78 (2008) 055016 [arXiv:0805.2623] [INSPIRE]. 